\title{
1 Pancreatic islet chromatin accessibility and conformation 2 defines distal enhancer networks of type 2 diabetes risk
}

4 William W Greenwald ${ }^{1,10}$, Joshua Chiou ${ }^{2,10}$, Jian Yan ${ }^{3,4,10}$, Yunjiang Qiu, ${ }^{1,30}$, Ning Dai ${ }^{5}$,

5 Allen Wang ${ }^{7}$, Naoki Nariai ${ }^{6}$, Anthony Aylward ${ }^{1}$, Jee Yun Han ${ }^{7}$, Nikita Kadakia ${ }^{6}$, Laura

6 Barrufet $^{5}$, Mei-Lin Okino ${ }^{6}$, Frauke Drees ${ }^{6}$, Nicholas Vinckier ${ }^{6}$, Liliana Minichiello ${ }^{8}$, David

7 Gorkin ${ }^{7}$, Joseph Avruch ${ }^{5}$, Kelly Frazer ${ }^{6,11}$, Maike Sander ${ }^{6,9,11}$, Bing Ren Ren $^{3,7,9,11}$, Kyle J

8 Gaulton $^{6,11, \#}$

10 1. Bioinformatics and Systems Biology Graduate Program, UC San Diego, La Jolla CA

11 2. Biomedical Sciences Graduate Program, UC San Diego, La Jolla CA

12 3. Ludwig Institute for Cancer Research, La Jolla CA

13 4. Department of Medical Biochemistry and Biophysics, Division of Functional

14 Genomics and Systems Biology, Karolinska Institutet, Stockholm, Sweden

15 5. Department of Molecular Biology, Harvard University, Cambridge MA

16 6. Department of Pediatrics, UC San Diego, La Jolla CA

17 7. Center for Epigenomics, UC San Diego, La Jolla CA

18 8. Department of Pharmacology, University of Oxford, Oxford UK OX1 3QT

19 9. Department of Cellular and Molecular Medicine, UC San Diego, La Jolla CA

20 10. Authors contributed equally to this work

21 11. Authors jointly supervised this work

22

23 \# Corresponding author: kgaulton@ucsd.edu 


\section{Abstract}

37 The gene targets of enhancer activity in pancreatic islets are largely unknown, impeding 38 discovery of islet regulatory networks involved in type 2 diabetes (T2D) risk. We 39 mapped chromatin state, accessibility and conformation using ChIP-seq, ATAC-seq and $40 \mathrm{Hi}-\mathrm{C}$ in human pancreatic islets, which we integrated with T2D genetic fine-mapping and 41 islet expression QTL data. Active islet regulatory elements preferentially interacted with 42 other active elements, often at distances over $1 \mathrm{MB}$, and we identified target genes for 43 thousands of distal islet enhancers. A third of T2D risk signals mapped in islet 44 enhancers, and target genes regulated by these signals were specifically involved in 45 processes related to protein transport and secretion. Among implicated target genes of 46 T2D islet enhancer signals with no prior known role in islet function, we demonstrated 47 that reduced IGF2BP2 activity in mouse islets leads to impaired glucose-stimulated 48 insulin secretion. These results link distal islet enhancer regulation of protein secretion 49 and transport to genetic risk of T2D, and highlight the utility of high-throughput chromatin 50 conformation maps to uncover the gene regulatory networks of complex disease.

\section{Introduction}

54 Genetic risk of type 2 diabetes (T2D) is largely mediated through variants affecting 55 transcriptional regulatory activity in pancreatic islets ${ }^{1-7}$. Genetic fine-mapping combined 56 with epigenomic annotation data can identify causal variants at T2D risk loci mapping in 57 islet regulatory elements ${ }^{1,2}$. The gene targets of islet regulatory elements, however, are 58 largely unknown, impeding discovery of disease-relevant gene networks perturbed by 59 risk variants and novel therapeutic avenues. The spatial organization of chromatin plays

60 a critical role in tissue-specific gene regulation, and recently developed high-throughput 61 techniques such as $\mathrm{Hi}-\mathrm{C}$ identify physical relationships between genomic regions in 62 human tissues genome-wide ${ }^{8-10}$. Tissue-specific maps of chromatin organization can 63 identify candidate target genes of distal regulatory elements and inform the molecular 64 mechanisms of disease risk variants ${ }^{9}$.

66 Here, we defined the spatial organization of transcriptional regulatory elements in 67 primary pancreatic islets, through which we mapped genetic effects on islet gene 68 expression and T2D risk. Islet active regulatory elements preferentially interacted with 
69 other active elements, in many cases over $1 \mathrm{MB}$, and we identified putative distal target

70 genes for thousands of islet enhancers. A third of known T2D risk signals had likely

71 causal variants in islet enhancers, and target genes of these signals were strongly

72 enriched for processes related to protein secretion and transport. Among target genes

73 with no previously known role in islet function, we demonstrated that reduced activity of

74 IGF2BP2 in mouse islets leads to impaired glucose-stimulated insulin secretion.

75 Together our results define distal regulatory programs in islets through which we link

76 islet enhancer regulation of protein transport and secretion to T2D risk.

\section{Results}

We first defined islet accessible chromatin using ATAC-seq ${ }^{11}$ generated from four

81 pancreatic islet samples (Table S1). Accessible chromatin signal was highly concordant

82 across all samples (Pearson $r^{2}>$.8) (Figure S1). We called sites for each sample

83 separately using MACS2 ${ }^{12}$, and merged sites to create a combined set of 105,734 islet

84 accessible chromatin sites. We then collected published ChIP-seq data of histone

85 modification and transcription factor binding in primary islets ${ }^{5,13}$ and called chromatin

86 states using ChromHMM ${ }^{14}$ (Figure S1). Accessible chromatin predominantly mapped

87 within active enhancer and promoter states (Figure 1A). We functionally annotated islet

88 accessible chromatin by using chromatin states to define active enhancers and

89 promoters, as well as other classes of islet regulatory elements (Table S2). We identified

9044,860 active enhancers which, in line with previous reports ${ }^{4,15}$, were distal to promoters,

91 more tissue-specific, and preferentially harbored motifs for FOXA, RFX, NEUROD and

92 other islet transcription factors (Figure S1, Table S3). These results define active

93 enhancers and other classes of regulatory elements in pancreatic islets.

95 Defining the target genes of enhancers has been challenging as they frequently control

96 non-adjacent genes over large genomic distances through chromatin looping ${ }^{16}$. The

97 spatial organization of chromatin in pancreatic islets is unknown, and we therefore

98 identified physical interactions between genomic regions in islets. We performed

99 genome-wide chromatin conformation capture using in situ $\mathrm{Hi}-\mathrm{C}^{8,17}$ in three islet

100 samples, two of which were sequenced to a depth of $>1$ billion reads (Table S1).

101 Contact matrices from islet $\mathrm{Hi}-\mathrm{C}$ assays were highly concordant across samples (Figure

102 S2). We called chromatin loops at $5 \mathrm{~kb}, 10 \mathrm{~kb}$, and $25 \mathrm{~kb}$ resolution with HICCUPS ${ }^{8}$ using 
103 reads from each sample individually, as well as with reads pooled from all three samples

104 (Figure 1B). We then merged loops across samples where both anchors overlapped at $10520 \mathrm{~kb}$ resolution (see Methods), resulting in a combined set of 11,924 islet Hi-C loops

106 (Table S4). The median distance between loop anchor midpoints was 255kb, and 107 nearly $10 \%$ were over $1 \mathrm{MB}$ in size (Figure S2). This established a map of chromatin 108 loops in human pancreatic islets.

110 We determined the relationship between islet regulatory element activity and chromatin 111 looping. Islet accessible chromatin signal was largely localized to islet loop anchors, 112 with the strongest signal at anchor midpoints (Figure 1C). Nearly half of all islet 113 regulatory elements were proximal to an anchor $(48.7 \%)$, and regulatory sites most 114 enriched (empirical $\mathrm{P}<1.5 \times 10^{-4}$ ) for chromatin loop anchors included CTCF binding sites 115 (2.61-fold), active promoters (2.08-fold), and active enhancers (1.85-fold) (Figure 1D).

116 We further mapped the relationship between islet regulatory sites connected by loop 117 anchors. The most significantly enriched anchor interactions were between active 118 enhancer and promoter sites (EnhA1-TssA OR=1.28, $\mathrm{P}=1.53 \times 10^{-37}$; EnhA1-EnhA1 $119 \mathrm{OR}=1.37, \mathrm{P}=1.87 \times 10^{-38}$; TssA-TssA OR=1.42, $\mathrm{P}=6.15 \times 10^{-36}$ ). We also observed strong 120 enrichment for interactions between CTCF binding sites (CTCF-CTCF OR=1.16; $121 \mathrm{P}=1.1 \times 10^{-17}$ ) (Figure 1E). These results demonstrate that islet chromatin loops are 122 prominently enriched for active regulatory sites in addition to CTCF binding sites.

124 We next used chromatin loops to annotate distal relationships between islet enhancers 125 and potential target genes genome-wide (see Methods). Over 40\% $(18,240)$ of islet 126 active enhancer elements interacted with at least one gene promoter region, and on 127 average, these enhancers interacted with 2 gene promoter regions (Figure S2, Table 128 S5). Conversely, the promoter regions of 8,448 genes had at least one loop to an 129 enhancer element (Figure 2A, Table S6). Of these 8,448 genes, 1,157 had more than 130 two independent chromatin loops to enhancer elements. Genes with multiple loops were 131 strongly enriched for processes related to transcription factor activity and gene 132 regulation, protein transport, and insulin signaling (Table S7). Among highly-looped 133 genes were also numerous critical for islet function, such as INS, ISL1, FOXA2, NKX6.1, 134 and $M A F B$ (Table S5). For example, there were four distinct loops between active 135 enhancers and $M A F B$, including several loops to enhancers over $1 \mathrm{MB}$ distal (Figure 136 2B). These results define candidate distal target genes of enhancer elements in islets. 
138 We examined the relationship between active enhancer interactions and target gene 139 expression level by using RNA-seq data from pancreatic islet samples ${ }^{18}$ and 53 tissues 140 in GTEx release $\mathrm{v} 7$ data $^{19}$. A significantly higher proportion of genes expressed in islets 141 had at least one enhancer loop compared to non-islet expressed genes $(\operatorname{In}(\mathrm{TPM})>1$; 142 expr=.48, non-expr=.30, Chi-square $\left.P=2.2 \times 10^{-16}\right)$. Genes with increasing numbers of 143 enhancer loops had, on average, higher expression level in islets $\left(\rho=.15, P=2.2 \times 10^{-16}\right)$, 144 with the highest expression among genes with 6 or more loops (median=19.8 TPM) 145 (Figure 2C). The number of islet enhancer interactions was also a significant predictor 146 of expression level in islets $\left(\beta=.10, P=6.4 \times 10^{-4}\right)$, and not of relative expression level in 147 any of the other 53 tissues in GTEx (all $P>$.05) (Figure 2D). We measured the relative 148 expression level of genes in islets and 53 GTEx tissues normalized across all tissues 149 (see Methods), and again observed a significant relationship between enhancer loops 150 and relative expression level in islets and no other tissues (Figure S2). These results 151 suggest distal islet enhancer chromatin loops are correlated with islet-specific gene 152 expression patterns.

154 We next determined the effects of genetic variants in islet enhancers on target gene 155 regulation. We generated expression quantitative trait loci (eQTL) data in 230 islet RNA156 seq samples by combining summary statistics from two published studies through meta157 analysis $^{7,18}$ (see Methods). We identified variants overlapping classes of islet regulatory 158 elements genome-wide. We then quantified the eQTL association of these variants to 159 target genes determined from their proximity to nearby genes and from chromatin loops 160 (see Methods). As expected, we observed the strongest eQTL evidence for active 161 promoter and enhancer variants proximal to genes (TssA median $-\log 10(P)=.65$, EnhA 162 proximal median $-\log 10(P)=.50$ ) (Figure 2E). For variants in distal enhancers, we 163 observed stronger evidence for islet eQTL association among genes in loops relative to 164 non-loop genes (EnhA interacting median=.35, EnhA non-interacting median=.31, 165 Wilcox $P=2.2 \times 10^{-16}$ ), even when matching based on gene distance to the enhancer 166 (Wilcox $\mathrm{P}=2.9 \times 10^{-4}$ ) (Figure 2E). These results suggest that genetic variants in distal 167 islet enhancer elements are preferentially correlated with the expression level of genes 168 within chromatin loops. 
170 Genetic variants at T2D risk loci are enriched for islet regulatory elements ${ }^{1,2,4,5}$, but the

171 effects of variants in regulatory elements on T2D risk in the context of chromatin looping

172 is unknown. We determined the effects of variants in islet regulatory elements and

173 chromatin loops on T2D risk using association data of $6.1 \mathrm{M}$ common (MAF>.05) variants

174 with fgwas and LD-score regression ${ }^{20,21}$. We observed strongest enrichment of variants

175 in active regulatory elements, most notably in active enhancers (EnhA1 fgwas

$176 \operatorname{In}$ (enrich)=3.9, LD-score Z=3.1) (Figure 3A, Figure S3). The effects of variants in active

177 enhancer and promoter elements on T2D risk were more pronounced among those in

178 chromatin loops (EnhA1 fgwas In(enrich)=4.38, LD-score Z=3.1; TssA fgwas

$179 \operatorname{In}$ (enrich)=3.03, LD-score Z=0.86) (Figure 3B, Figure S3). Conversely, variants in other

180 islet elements such as flanking promoters and weak enhancers and were more enriched

181 outside of loops (Figure 3B, Figure S3). To determine the inter-dependence of these

182 effects, we jointly modelled variants in islet regulatory elements on T2D risk, while also

183 including variants in GENCODE coding exons and UTRs. In a joint model, we observed

184 enrichment of variants in islet active enhancer elements (EnhA1 In(enrich)=4.04), in

185 addition to flanking promoters (TssFInk In(enrich)=3.77) and coding exons (CDS

186 In(enrich)=2.34) (Figure S3). These results demonstrate genome-wide enrichment of

187 variants in islet active regulatory elements within chromatin loops for T2D risk.

189 To identify T2D risk signals mapping in islet enhancers, we used effects from the joint 190 enrichment model as priors on the causal evidence (PPA) for both variants at known

191 T2D loci and in windows genome-wide ${ }^{1,2,21}$ (see Methods). Among 107 known risk

192 signals, variants in islet enhancers accounted for almost a third (29\%) of the total 193 probability mass (Table S8, Figure 3C). We clustered known risk signals based on

194 annotations at candidate causal variants (see Methods) and identified 30 signals where

195 the causal variant was likely in an islet enhancer (Figure 3D). The 30 T2D islet enhancer

196 signals were associated with IGTT-based insulin secretion phenotypes significantly more

197 than un-annotated signals ( $E n h=42 \%$, un-annot $=17 \%$, Chi-square $P=1 \times 10^{-7}$ ), supporting

198 a role in islet function ${ }^{22}$ (Figure 3E). Fine-mapping including functional priors improved

199 resolution of causal variants at the $30 \mathrm{~T} 2 \mathrm{D}$ islet enhancer signals (avg. 3.5 enh variants)

200 (Figure 3F), and at 6 signals resolved a single causal islet enhancer variant (Table S8).

201 One example is previously unreported variant rs7732130 (ZBED3; PPA=98\%) in a

202 chromatin loop and which has allelic effects on islet enhancer activity (T-test Fwd

$203 \mathrm{P}=3.7 \times 10^{-3}$, Rev $\mathrm{P}=6.8 \times 10^{-6}$ ) (Figure 3G, 3H). Outside of known loci, we identified an 
204 additional 131 1MB windows genome-wide harboring putative T2D enhancer variants

205 (Figure S3, Table S9; see Methods). These results identify a large number of known

206 and putative T2D risk signals with causal variants in islet enhancers.

208 A large percentage of T2D risk signals map in islet enhancers, and the gene targets of 209 these signals are largely unknown. We defined candidate target genes based on gene 210 promoter regions in chromatin loops with, or in proximity to, T2D enhancer signals

211 (Figure 3D, see Methods). T2D enhancer signals had on average 2 target genes 212 (Figure 4A, 4B, Table S10), a large reduction in candidate gene numbers obtained 213 when using a 1MB window (avg.=18 genes) or TAD definitions (avg.=7 genes) (Figure 214 4A). Target genes were enriched in gene sets related to protein transport and secretion, 215 potassium ion transport, vesicles and vesicle membranes, and endoplasmic reticulum 216 (FDR<.2) (Figure 4B, Table S11). Target genes also included multiple involved in 217 MODY and other monogenic and syndromic diabetes (ABCC8, KCNJ11, GCK, INS, 218 GLIS3, WFS1) (Figure S4). Conversely, non-target genes within 1MB of these same 30 219 signals were enriched for gene sets related to stress-response and other processes 220 (FDR<.2), suggesting regulatory programs potentially activated in other cellular states 221 (Table S11, see Methods). At several loci, loops implicated target genes highly distal 222 (>500kb) to T2D enhancer variants; for example, multiple KCNQ1 signals interacted with 223 INS/IGF2 over $700 \mathrm{~kb}$ distal, and ZMIZ1 interacted with POLR3A over $1 \mathrm{MB}$ distal (Figure 224 S4). These results define putative target genes of $T 2 D$ enhancer signals involved in 225 protein transport and secretion and monogenic diabetes.

227 We then further identified target genes regulated by T2D enhancer signals using islet 228 eQTL data. At each signal, we tested the most likely casual enhancer variant for eQTL 229 association to each target gene correcting for the total number of target genes (see 230 Methods). For genes with eQTL evidence $(\mathrm{P}<.05)$, we further confirmed eQTL and T2D 231 signals were unlikely to be driven by distinct causal variants using Bayesian co232 localization (see Methods). Target genes showed evidence of islet eQTLs with 8 known 233 T2D islet enhancer signals $(\mathrm{P}<.05)$ including $C A M K 1 D, A B C B 9, C 2 C D 4 B$, and IGF2BP2 234 (Figure 4D, Table S12). For example, known T2D variant rs11257655 is in an islet 235 active enhancer element that loops to the CAMK1D promoter and is an islet eQTL for 236 CAMK1D expression ${ }^{23}$ (Figure 4E). At the 131 putative T2D enhancer signals, we 237 identified 12 additional target genes with evidence for eQTLs to T2D variants $(P<.05)$ 
238 such as FADS1, VEGFA, SNX32 and SCRN2 (Table S12). Among the 21 directly

239 regulated genes, nearly a third have not been identified as significant islet eQTLs in

240 previous studies ${ }^{7,18,41}$. Target genes with islet eQTLs to known and putative T2D

241 enhancer signals were specifically enriched for genes involved in vesicle-mediated

242 transport (FDR<.2) (Figure 4C, Table S11). These results demonstrate that target

243 genes of T2D islet enhancer signals are involved in protein transport and secretion.

245 Among novel target genes, IGF2BP2 has a strong islet eQTL with T2D enhancer 246 variants and has no known role in T2D-relevant islet biology. As T2D risk alleles are 247 correlated with reduced IGF2BP2 expression and reduced insulin secretion 248 phenotypes ${ }^{22}$, we hypothesized that reduced IGF2BP2 expression in islets would 249 increase T2D risk. We thus determined the effects of reduced IGF2BP2 on islet function 250 using a mouse model. IGF2BP2/Imp2 is widely expressed in adult mouse tissues 251 including fat, muscle, liver and pancreas $^{24}$, and in the pancreas Imp2 expression 252 localized to islets and overlapped insulin (Figure 5A). We inactivated Imp2 in mouse 253 beta cells by recombining the $\operatorname{Imp} 2^{\text {flox(f) }}$ allele with Cre recombinase driven by the rat 254 insulin 2 promoter (RIP2-Cre) (Figure S5A). Immunoblot analysis of extracts from 255 isolated $I m p 2^{f f} / R I P 2-C r e$ islets confirmed reduced Imp2 abundance compared to Imp2 ${ }^{\text {ff }}$

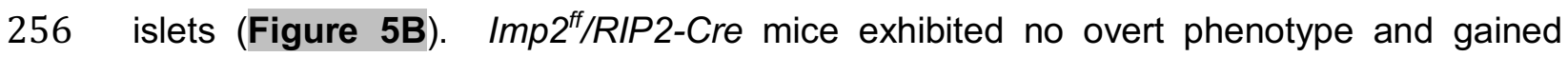
257 weight similar to Imp2 $2^{\text {ff }}$ controls on both a normal chow (NCD) and high fat diet (HFD) 258 (Figure S5B).

260 We next assessed the effect of IGF2BP2 deficiency in mouse beta cells on glucose 261 homeostasis. At 10 weeks of age, Imp2 $2^{f f}$ and $I m p 2^{f f} / R I P 2-C r e$ mice on NCD exhibited no 262 difference in blood glucose and insulin levels. By contrast, blood insulin and C-peptide 263 levels were reduced in HFD-fed Imp2 /RIP2-Cre compared to HFD-fed control mice, 264 whereas blood glucose and glucagon levels were similar (Figure 5C). When challenged 265 with an intraperitoneal glucose injection, HFD-fed, but not NCD-fed, Imp2 ff/RIP2-Cre 266 mice exhibited significantly higher glucose and lower insulin levels than Imp2 $2^{\text {ff }}$ mice 267 (Figure 5D,E). Importantly, this was not due to a difference in insulin sensitivity, as blood 268 glucose levels after an intraperitoneal insulin injection were similar in $I m p 2^{\text {ff }}$ and $269 \mathrm{Imp} 2$ ff/RIP2-Cre mice (Figure S5C). These results indicate that IGF2BP2 deficiency 270 limits the capacity of beta cells to augment insulin secretion in response to increased 271 insulin demand. 
273 In summary, we defined the genomic location, function, and spatial orientation of

274 regulatory elements in pancreatic islets. Islet active regulatory elements preferentially

275 interacted with other active elements, in many cases at distances over $1 \mathrm{MB}$, and we

276 identified putative target genes for thousands of islet distal enhancers. Target genes of

277 T2D islet enhancer signals were specifically involved in processes related to protein

278 transport and secretion, and we validated that reduced activity of a previously unknown

279 target gene IGF2BP2 in mouse islets leads to defects in glucose-stimulated insulin 280 secretion. Together our results define distal regulatory networks in islets and link T2D 281 risk to enhancer regulation of protein transport and secretion. Furthermore, these 282 results highlight the utility of high-resolution chromatin conformation maps in dissecting 283 the gene regulatory networks underlying genetic risk of T2D and other complex disease.

\section{Methods}

\section{Islet ATAC-seq data generation}

288 Four human islet donors were obtained from the Integrated Islet Distribution Program 289 (IIDP) (Table S1). Islet preparations were further enriched and selected using zinc290 dithizone staining. We generated ATAC-seq data from the four human islet samples 291 with a protocol as previously described ${ }^{11}$. For each sample, we trimmed adaptor 292 sequences using TrimGalore (https://github.com/FelixKrueger/TrimGalore). The resulting 293 sequences were aligned to sex-specific hg19 reference genomes using bwa mem ${ }^{25,26}$. 294 We filtered reads were to retain those in proper pairs and with mapping quality score 295 greater than 30. We then removed duplicate and non-autosomal reads. We called 296 peaks individually for each sample with MACS2 ${ }^{12}$ at a q-value threshold of .05 with the 297 following options "-no-model”, “- shift -100”, “-extsize 200”. We removed peaks that 298 overlapped genomic regions blacklisted by the ENCODE consortium and merged the 299 peaks $^{26}$. In total, we obtained 105,734 merged peaks. To assess concordance between 300 ATAC-seq experiments, we calculated read coverage at $200 \mathrm{bp}$ bins genome-wide, 301 excluding blacklisted genomic regions. We then calculated the Pearson correlation 302 between the read counts for each sample. 
305 We generated Hi-C data from three pancreatic islet samples, two of which also had

306 ATAC-seq data (Table S1). Islet preparations were further enriched and selected using

307 zinc-dithizone staining. In situ $\mathrm{Hi}-\mathrm{C}$ was performed using a previously published protocol

308 with modifications adapted to frozen human tissue ${ }^{17}$. Briefly, the tissue was cut to fine

309 pieces and washed with cold PBS. Cross-linking was carried out with $1 \%$ formaldehyde

310 (sigma) in PBS at room temperature (RT) for 10 min and quenched with 125mM Glycine

311 (sigma) at RT for $5 \mathrm{~min}$. Nuclei were isolated using a loose-fitting douncer in hypotonic

312 buffer (20mM Hepes pH7.9, 10mM KCl, 1mM EDTA, 10\% Glycerol and 1mM DTT with

313 additional protease inhibitor (Roche) for 30 strokes and centrifuge at $4{ }^{\circ} \mathrm{C}$.

314

315 Nuclei were digested using 4 cutter restriction enzyme Mbol (NEB) at $37{ }^{\circ} \mathrm{C}$ overnight

316 (o/n). Digested ends were filled in blunt with dBTP with biotinylated-14-ATP (Life

317 Technologies) using Klenow DNA polymerase (NEB). Re-ligation was performed in situ

318 when nucleus was intact using T4 DNA ligase (NEB) at $16{ }^{\circ} \mathrm{C}$ for $4 \mathrm{hrs}$. The cross-linking

319 was reversed at $68^{\circ} \mathrm{C} \mathrm{o} / \mathrm{n}$ while protein was degraded with proteinase $\mathrm{K}$ treatment

320 (NEB). DNA was purified with phenol-chloroform extraction and ethanol precipitation,

321 followed by fragmentation to $300-500$ bp with the Covaris S220 ultrasonicator. Ligation

322 products were enriched with Dynabeads My One T1 Streptavidin beads (Life

323 Technologies). PCR was used to amplify the enriched DNA for sequencing. HiSeq 4000

324 sequencer (Illumina) was used to sequence the library with 2x100 bp paired-end reads.

326 For each sample, reads from paired end reads were aligned with bwa mem ${ }^{27}$ as single327 end reads, and then filtered through following steps. First, only five prime ends were kept

328 for chimeric reads. Second, reads with low mapping quality $(<10)$ were removed. Third, 329 read ends were then manually paired, and PCR duplicates were removed using Picard 330 tools (https://github.com/broadinstitute/picard). Finally, filtered contacts were used to 331 create chromatin contact maps with Juicebox ${ }^{28}$.

333 Contact maps for each sample were binned to $100 \mathrm{~kb}$, and the correlation between 334 samples across all bins for all chromosomes was calculated using scipy.stats.pearsonr 335 in scipy. Chromatin loops were identified by using HICCUPS $^{8}$ at $5 \mathrm{~kb}, 10 \mathrm{~kb}$, and $25 \mathrm{~kb}$ 336 resolutions with default parameters on the $\mathrm{Hi}-\mathrm{C}$ maps for each individual. The Hi-C data 337 was then pooled across all three samples to create a single contact map, and loops 338 were called with HICCUPs at the same resolutions with the same parameters. A single 
339 loop set was then created by identifying loops where both anchors were within 20kb of

340 one another via pgltools ${ }^{29}$ and retaining the loop with the highest resolution. If multiple

341 loops were found at the highest resolution, loops were kept from the contact map with

342 the highest overall sequencing depth. We also called topologically associated domains

343 (TADs) from the pooled $\mathrm{Hi}-\mathrm{C}$ data using a previously described approach $^{10}$.

\section{Islet ChIP-seq data processing}

346 We obtained previously published data from ChIP-seq assays of H3K4me1, H3K27ac,

347 H3K4me3, H3K36me3 and CTCF generated in primary islets and for which there was 348 matching input sequence from the same sample ${ }^{4-6}$. For each assay and input, we 349 aligned reads to the human genome hg19 using bwa ${ }^{30}$ with a flag to trim reads at a 350 quality threshold of less than 15. We converted the alignments to bam format and 351 sorted the bam files. We then removed duplicate reads, and further filtered reads that 352 had a mapping quality score below 30 . Multiple sequence datasets obtained from the 353 same assay in the same sample were then pooled.

355 We defined chromatin states from ChIP-seq data using ChromHMM ${ }^{14}$ with a 9-state 356 model, as calling larger state numbers did not empirically appear to identify additional 357 states. We assigned the resulting states names based on patterns previously described 358 in the NIH Roadmap and ENCODE projects - CTCF (CTCF), Transcribed (Txn; 359 H3K36me3), Active promoter (TssA; H3K4me3, H3K4me1), Flanking promoter (TssFInk; 360 H3K4me3, H3K4me1, H3K27ac), Weak/Poised Enhancer (EnhWk; H3K4me1), Active 361 Enhancer 1 (EnhA-1; H3K27ac), Active Enhancer 2 (EnhA-2; H3K27ac, H3K4me1), and 362 two Quiescent states (Quies; low signal for all assays).

364 We then annotated accessible chromatin sites based on overlap with the chromatin 365 states. If an accessible chromatin site overlapped multiple chromatin states, we split the 366 site into multiple distinct elements.

\section{Islet chromatin interaction analyses}

369 To determine the normalized tag counts of ATAC-seq data at loop anchors, loop anchors 370 were converted to a regular BED file with pgltools ${ }^{29}$, and $\mathrm{HOMER}^{31}$ was used to find the 371 normalized tag density across all loop anchors for each ATAC-seq sample. Output from 372 HOMER was normalized to a maximum height of 1 for each sample to determine the 
373 distribution of ATAC-seq signal within each sample, rather than the relative magnitude

374 coverage difference between ATAC-seq samples.

375

376 To determine the enrichment of each class of islet regulatory elements near loops, and

377 the types of elements colocalized by loops, we utilized pgltools and HOMER to integrate

378 the ATAC-seq and $\mathrm{Hi}-\mathrm{C}$ data. We first created a size matched null distribution

379 comprised of 7,000 permuted regions. Next, for each islet accessible chromatin state,

380 we identified the proportion of states within $25 \mathrm{~kb}$ of a loop. We determined the fold

381 enrichment of each class over the average calculated from the null distribution, and

382 determined significance as the number of permuted counts greater than the observed.

383

384 To determine which pairs of islet regulatory elements were in chromatin loops at a

385 statistically significant level, we compared the distribution of islet regulatory elements

386 around loop anchors using HOMER. We utilized the "annotatelnteractions" function to

387 obtain logistic regression $p$-values and odds ratio enrichment estimates for all pairs of

388 islet regulatory elements.

390 We defined candidate target genes of islet enhancer elements using Hi-C loops in the 391 following way. First, we identified all islet active enhancer elements mapping within 25kb

392 of a Hi-C loop anchor. We then filtered these loops based on whether the other anchor 393 mapped within $25 \mathrm{~kb}$ of a promoter region $(-5 \mathrm{~kb} /+2 \mathrm{~kb}$ of transcription start site) for 394 protein-coding or long non-coding genes in GENCODEv27 $7^{32}$. For each active enhancer, 395 we then calculated the number of gene promoter regions interacting with that enhancer. 396 For each gene promoter region, we calculated the number of independent interactions 397 containing at least one active enhancer element.

We identified genes with multiple $(>2)$ active enhancer interactions and tested these 400 genes for gene set enrichment using GSEA $^{33}$, considering only gene sets with more than 40125 genes at an FDR>.2.

\section{Genomic enrichment analyses}

404 We tested for enrichment of variants in each accessible chromatin class using T2D 405 association data of 1000 Genomes project variants from the DIAGRAM consortium ${ }^{21}$. 406 From this meta-analysis, we identified common variants (with minor allele frequency 
407 (MAF)>.05). In total, we retained 6.1M common variants for testing. For each variant,

408 we then calculated a Bayes Factor from effect size estimates and standard errors using

409 the approach of Wakefield ${ }^{34}$.

411 We then modelled the effect of variants in each class of islet regulatory elements on T2D

412 risk using fgwas $^{20}$. For these analyses, we used a window size (-k) that resulted in a

$4131 \mathrm{Mb}$ window on average. We first tested for enrichment of variants in each state

414 individually. We then built a joint model iteratively in the following way. We first identified

415 the annotation with the highest likelihood. We then added annotations to the model until

416 the likelihood did not increase further. Using this model, we introduced a series of

417 penalties from 0 to .5 in increments of .01 and fit the model using each penalty, and

418 identified the penalty that gave the highest cross-validation likelihood. We then finally

419 removed annotations from the model that further increased the cross-validation

420 likelihood. We considered the resulting set of annotations and effects to be the optimal

421 joint model.

423 We also modelled the effect of variants in islet regulatory elements using LD-score

424 regression. For these analyses, we extracted variants in HapMap3 from T2D

425 association data. We then calculated LD scores for variants in each regulatory element

426 class. Finally, we obtained enrichment estimates using these LD scores with T2D

427 association data of HapMap3 variants.

\section{Fine-mapping of T2D risk variants}

430 We used the effects from the joint enrichment model as priors on the evidence for 431 variants at 107 known T2D signals using fine-mapping data from the Metabochip ${ }^{2}$, 432 GoT2D ${ }^{1}$ and DIAGRAM 1000 Genomes $^{21}$ studies. We used data of 49 T2D signals at 43339 T2D loci on the Metabochip, 41 additional T2D signals from GoT2D data for T2D loci 434 not on the Metabochip, and 17 additional T2D signals in DIAGRAM 1000G not in 435 Metabochip or GoT2D.

437 For each signal, we obtained the enrichment effect of the islet regulatory or coding 438 annotation overlapping that variant. We calculated a prior probability for the variant by 439 dividing the effect by the sum of effects across all variants at a signal. We then 440 multiplied this prior probability by the Bayes Factor for each variant. From the resulting 
441 odds, we calculated a posterior probability that the variant is causal for T2D risk (PPA)

442 by dividing the odds by the sum of odds across all variants at the locus.

444 For each signal, we calculated a cumulative PPA (cPPA) value for islet enhancer 445 (EnhA1, EnhA2, EnhWk), promoter (TssA, TssFlnk), CTCF binding site, UTR, and 446 coding exon (CDS) annotations by summing the PPAs of all variants overlapping each 447 annotation. We then clustered T2D signals into groups based on cPPA values using k448 means clustering.

450 We determined the effects of T2D signals in each cluster on glycemic association data ${ }^{22}$. 451 We identified 73 T2D signals represented in these data and cataloged 23 associated at $452 \mathrm{P}<.05$ with first-phase insulin response, peak insulin response, AIR, or insulin secretion 453 rate. We calculated the percentage of signals in each cluster associated with these 454 measures and tested for differences between clusters using a chi-square test.

456 For the 30 T2D islet enhancer signals, we calculated "99\% credible sets" as the set of 457 candidate variants that explain $99 \%$ of the total PPA using genetic fine-mapping data 458 alone (genetic), and fine-mapping including priors from the joint genome-wide 459 enrichment model (+priors).

461 We then fine-mapped casual variants in putative T2D loci genome-wide. For variants in 462 each $1 \mathrm{MB}$ window across the genome, after excluding any windows overlapping a 463 known T2D signal, we obtained the effect of the islet annotation overlapping that variant.

464 We calculated a prior probability for each variant as described above also including an 465 additional prior on the evidence that the $1 \mathrm{MB}$ window is a T2D locus. We multiplied both 466 prior probabilities by the Bayes Factor for each variant. From the resulting odds, we 467 calculated the PPA that each variant is causal for T2D risk. We then considered the 131 468 windows with at least one islet enhancer variant with PPA>.01 in downstream analyses.

\section{Genomic features analyses}

471 For each class of islet open chromatin, we determined the overlap with other genomic 472 features. 
474 We identified motifs enriched in sequence underneath each islet accessible chromatin

475 class. We first extracted genomic sequence for each site using bedtools ${ }^{35}$, and masked

476 repetitive sequences. We then identified de novo motifs enriched in this sequence using

$477 \mathrm{DREME}^{36}$. For each de novo motif, we determined whether this motif matched a known

478 sequence motif in a custom database of $>2,500$ motifs from ENCODE, JASPAR and

479 SELEX with tomtom ${ }^{26,37-39}$.

481 We determined the overlap of islet accessible chromatin classes with transcription factor 482 (TF) ChIP-seq data in islets for 5 proteins $^{4,26}$. For each islet chromatin class, we 483 calculated the Jaccard index of overlap with sites for each $\mathrm{TF}^{35}$. We then determined 484 the overlap of islet accessible chromatin classes with DHS sites from 384 cell types in 485 the ENCODE project ${ }^{26}$. We first filtered out DHS sites from islets, and then for each 486 accessible chromatin site, we calculated the percentage of ENCODE cell-types the site 487 was active in. We then determined the median percent overlap across all sites within 488 each accessible chromatin class.

\section{Gene expression analysis}

491 We obtained transcript-per-million (TPM) counts from RNA-seq data in 53 tissues from 492 the GTEx project release $v 7^{19}$. We further obtained RPKM read counts from RNA-seq 493 data of 118 pancreatic islet samples ${ }^{18}$, and calculated TPM values as previously 494 described ${ }^{40}$. We then retained only protein-coding and long non-coding genes 495 annotated in GENCODEv2 $7^{32}$. We first calculated the percentage of genes expressed in 496 islets (defined as $\ln ($ TPM $>>1$ ) and not expressed in islets with at least one enhancer 497 chromatin loop to the promoter region and tested for a significant difference using a Chi498 square test.

500 Across all 54 tissues, we filtered out genes not expressed $(\ln (T P M)>1)$ in at least one 501 tissue. We determined correlation between gene expression level in islets and enhancer 502 loop number using Spearman's rho. We further grouped genes by the number of 503 chromatin loops to enhancer elements and calculated the median islet TPM value for 504 each group. For genes with at least one enhancer loop, we created a linear model of 505 log-transformed gene TPM with chromatin loop number as the predictor using the glm 506 package in R. Values are reported as the p-value, effect size (beta) and standard error 507 from the resulting model. 
509 We then determined the relative expression level for each gene in 54 tissues. We log-

510 transformed expression values and calculated a z-score for each gene using the mean

511 and standard deviation across tissues. We then repeated the above analyses using

512 tissue z-scores instead of tissue TPM values.

513

\section{Islet expression QTL analysis}

515 We obtained summary statistic eQTL data from two published studies of 118 and 112 516 primary pancreatic islet samples ${ }^{18,41}$. We then performed inverse sample-size weighted 517 meta-analysis to combine the summary results for each variant and gene pair using $518 \mathrm{METAL}^{42}$. We retained only protein-coding and long non-coding RNA genes as defined 519 by GENCODEv $27^{32}$, only variant and gene pairs tested in both studies, and only variants 520 with minor allele frequency $(\mathrm{MAF})>.01$.

521

522 We extracted eQTL associations for variants in classes of islet accessible chromatin. To 523 remove potential biases due to linkage disequilibrium, we sorted variant associations 524 based on $p$-value and iteratively pruned out variants in $L D\left(r^{2}>.5\right)$ with a more significant 525 variant using LD information in European samples from 1000 Genomes project data. 526 We then extracted pruned eQTL associations for variants in active promoter elements 527 for genes within $20 \mathrm{~kb}$ (TssA), variants in active enhancer elements for genes within 20 $528 \mathrm{~kb}$ (EnhA proximal), variants in active enhancer elements for genes in chromatin loops 529 (EnhA distal target), and variants in active enhancer elements for genes without a loop 530 (EnhA distal no-target). For each set of eQTL associations, we compared p-value 531 distributions using a two-sided Wilcox rank-sum test. To remove potential biases in 532 variant distances to loop and no-loop genes, we randomly selected variant-gene pairs 533 matched on distance to the distal target set to re-performed analyses.

\section{Target genes of T2D islet enhancer signals}

536 We defined candidate target genes of 30 known T2D enhancer signals and 131 putative 537 T2D enhancer windows in the following way. We identified candidate causal variants at 538 each signal overlapping islet enhancer elements and considered target genes as those 539 where a candidate variant either (a) mapped in a chromatin loop to the promoter region $540(-5 \mathrm{~kb} /+2 \mathrm{~kb}$ of transcription start site) or (b) was within $25 \mathrm{~kb}$ proximal to the promoter 541 region. 
543 We next defined alternate sets of target genes of the $30 \mathrm{~T} 2 \mathrm{D}$ enhancer signals based on

$5441 \mathrm{MB}$ windows or TAD boundaries. For 1MB window definitions, we identified the

545 highest probability variant for each signal and extracted a $+/-1 \mathrm{MB}$ window around the

546 variant position. We then considered gene promoter regions $(-5 \mathrm{~kb} /+2 \mathrm{~kb}$ of transcription

547 start site) for protein-coding or long non-coding genes in GENCODEv27 that overlapped

548 this $+/-1 \mathrm{MB}$ window the set of target genes. For TAD boundary definitions, we

549 intersected the merged set of TADs with gene promoter regions to obtain a set of genes

550 within each TAD. We then intersected the highest probability variant at each T2D signal

551 with TADs to obtain gene sets in the TAD.

552

553 For each enhancer signal with a candidate target gene, we extracted eQTL $p$-values for

554 each target gene using the islet enhancer variant with the highest PPA at the signal.

555 Where the highest probability variant was not present in the eQTL dataset, we used the

556 next most probable islet enhancer variant. We then Bonferroni-corrected eQTL $p$-values

557 for the total number of candidate target genes at the signal and considered eQTLs

558 significant with a corrected $p$-value<.05.

559

560 For genes with significant eQTL evidence we further tested whether T2D and eQTL 561 signals were co-localized. We obtained the T2D Bayes Factor for each variant at the 562 signal from fine-mapping data. For significant gene eQTLs at the signal, we then 563 calculated the Bayes Factor that each variant is an islet eQTL for that gene ${ }^{34}$. We 564 compared Bayes Factors for T2D signals and eQTLs for each gene using Bayesian co565 localization ${ }^{43}$. We considered the prior probability that a variant was causal for T2D risk 566 or an islet eQTL as $1 \times 10^{-4}$, and the prior probability that a variant was causal for both 567 T2D risk and an islet eQTL as $1 \times 10^{-5}$. We considered signals as having evidence for co568 localization where the probability of a shared causal variant was higher than the 569 probability of two distinct causal variants.

570

571 We tested target genes for gene set enrichment using GSEA ${ }^{33}$, considering only gene 572 sets with more than 25 total genes and at an FDR threshold of 2 .

573

\section{$574 \quad$ Luciferase reporter assays}


575 To test for allelic differences in enhancer activity at rs7732130, we cloned sequences

576 containing alt or ref alleles in forward and reverse orientation upstream of the minimal

577 promoter of firefly luciferase vector pGL4.23 (Promega) using Kpnl and Sacl restriction

578 sites.

579

580 The primer sequences were:

581 forward/left: GATAACGGTACCGCGAAGTGGTCATGGGTAAA

582 forward/right: AAGTAGGAGCTCACCATCCCAGCATTTAGTGG

583 reverse/left: GATAACGAGCTCGCGAAGTGGTCATGGGTAAA

584 reverse/right: AAGTAGGGTACCACCATCCCAGCATTTAGTGG

585

586 MIN6 beta cells were seeded into 6 (or 12)-well trays at 1 million cells per well. At $80 \%$ confluency, cells were co-transfected with 400ng of the experimental firefly luciferase vector pGL4.23 containing the alt or ref allele in either orientation or an empty vector and $50 \mathrm{ng}$ of the vector pRL-SV40 (Promega) using the Lipofectamine 3000 reagent. All transfections were done in triplicate. Cells were lysed 48 hours after transfection and assayed for Firefly and Renilla luciferase activities using the Dual-Luciferase Reporter

592 system (Promega). Firefly activity was normalized to Renilla activity and compared to the

593 empty vector and normalized results were expressed as fold change compared to empty 594 vector control per allele. Error bars are reported as standard deviation. A two-sided t595 test was used to compare luciferase activity between the two alleles in each orientation.

\section{Mouse Imp2 targeting construct and physiological studies}

598 We generated the Imp2 construct by using a genomic fragment of $12 \mathrm{~kb}$ containing Imp2

599 exons 1 and 2 as well as flanking intron sequences of the murine gene extracted from 600 the RP23-163F16 BAC clone. The replacement-type targeting construct consisted of 9.4 $601 \mathrm{~kb}$ of Imp2 genomic sequences (4.4 kb in the left homology arm and $5.4 \mathrm{~kb}$ in the right 602 homology arm) (Figure S5).

604 We bred mice for experiments by crossing IMP2-loxp mice (Imp2 ${ }^{\text {ff }}$ ) with RIP2-Cre mice 605 on a C57BI/6J background. We maintained colonies in a specific pathogen-free facility 606 with a 12:12 light - dark cycle and fed irradiated chow (Prolab 5P75 Isopro 3000; 5\% 607 crude fat; PMI nutrition international) or a HFD (D12492i; 60\% kcal fat; Research Diets 608 Inc.). Blood glucose, insulin, C-peptide and glucagon levels were measured by the 
609 Vanderbilt metabolic core. Measurements for $I m p 2^{f f}$ and $I m p 2^{f f} / R I P 2-C r e$ mice were

610 performed using male mice under basal conditions $(\mathrm{N}=10)$, upon intraperitoneal glucose

611 injection ( $N=9)$, and upon intraperitoneal insulin injection ( $N=9)$. A two-sided t-test was

612 used to compare differences in measurements across genotypes.

613

\section{Acknowledgements}

615 Support for this work was provided by U01DK105541 to M.S., K.F. and B.R., the Ludwig

616 Institute for Cancer Research to B.R., R37DK017776 and P30DK057521 to J.A.,

617 postdoc fellowship 537-2014-6796 from the Swedish Vetenskapsrådet to J.Y., and

618 JDRF-3-2012-177 postdoc fellowship to A.W.

619

620 Author Contributions

621 K.J.G., B.R., M.S., K.F. conceived of and supervised the research in the study; K.J.G.

622 wrote the manuscript and performed analyses; W.W.G, J.C., Y.Q. performed analyses

623 and contributed to writing; J.Y. performed Hi-C assays and contributed to writing; N.D.,

624 J.A. performed mouse experiments and contributed to writing; A.W., A.A. contributed

625 analyses and data interpretation; J.Y.H., N.V., F.D., D.G. performed ATAC-seq assays

626 and contributed to data interpretation; N.K. and M.O. performed variant reporter

627 experiments; L.B. and L.M. contributed to mouse experiments.

628

629 Data availability

630 Data files for this study are available at http://gaultonlab.org/pages/Greenwald_islet_HiC

631 and will also be deposited in https//www.t2depigenome.org 


\section{Figure legends}

634

\section{Main Figures:}

637 Figure 1. Chromatin accessibility and conformation in pancreatic islets. (A) Islet 638 accessible chromatin signal mapped predominantly within active islet chromatin states. 639 (B) Chromatin looping from in situ Hi-C assays of three pancreatic islet samples at entire 640 chromosome (left), 25MB (middle) and 2MB (right) resolution on chromosome 7. Black 641 circles on the right panel represent statistically significant loop calls. (C) Accessible 642 chromatin signal from four islet samples (ISL1-4) was distributed around chromatin loop 643 anchor midpoints (D) Islet chromatin loop anchors were enriched for islet CTCF binding 644 sites, active enhancers, and active promoters compared to random sites. Values 645 represent fold change and SD. (E) Islet chromatin loops were most enriched for 646 interactions between islet active enhancers and active promoter elements, and between 647 CTCF binding sites.

Figure 2. Islet enhancer regulation of distal target gene expression. (A) The 650 promoter regions of $8.4 \mathrm{k}$ genes had at least one chromatin loop to an islet enhancer 651 element. (B) Multiple islet enhancers formed chromatin loops with the MAFB promoter 652 region including several over $1 \mathrm{MB}$. (C) Genes with increasing numbers of chromatin 653 loops to islet enhancers had increased expression level in islets, with the highest 654 expression among genes with 6 or more interactions (D) The number of chromatin loops 655 to islet enhancers was a significant predictor of islet gene expression but not 53 other 656 tissues in GTEx. Values represent effect size and SE. ${ }^{* *} \mathrm{P}<.001$ (E) Genetic variants in 657 distal islet enhancer elements had stronger evidence for islet expression QTLs with 658 genes in chromatin loops (blue) than genes with no loop (grey), even when matched 659 based on distance (light blue). ${ }^{* *} \mathrm{P}<.001,{ }^{* *} \mathrm{P}<.0001$

661 Figure 3. Type 2 diabetes risk signals map in islet enhancers. (A) Genetic variants 662 in islet active regulatory elements genome-wide were enriched for T2D risk, with 663 strongest enrichment in active enhancer elements. Values represent log enrichment and $66495 \% \mathrm{Cl}$. (B) The effects of variants in active enhancer and promoter elements on T2D 665 risk were stronger among those in chromatin loops, whereas other elements were 666 enriched for T2D outside of loops. Values represent log enrichment and $95 \% \mathrm{Cl}$. (C) 
667 Over $30 \%$ of the total causal probability across 107 known T2D risk signals mapped in

668 islet enhancer elements. (D) Clustering of known T2D signals based on islet and coding 669 annotations identified 30 signals with likely causal variants in islet enhancers. (E) A 670 significantly higher percentage of T2D islet enhancer signals were associated with IGTT671 based insulin secretion phenotypes than un-annotated T2D signals. ${ }^{* *} P<.001(F)$ 672 Number of variants in the $99 \%$ credible sets for the 30 T2D islet enhancer signals based 673 on genetic fine-mapping alone (genetic), genetic fine-mapping including functional priors 674 (+priors) (G) T2D causal variant rs7732130 at the ZBED3 locus mapped in an islet active 675 enhancer and chromatin loop, and had $(\mathrm{H})$ allelic effects on enhancer activity in the islet 676 cell line MIN6 $(\mathrm{N}=3)$. Values represent fold change and SD. ${ }^{* *} \mathrm{P}<.001,{ }^{* * *} \mathrm{P}<.0001$

678 Figure 4. Target genes of type 2 diabetes islet enhancer signals are involved in 679 protein secretion and transport. (A) Prioritizing target genes using chromatin loops 680 and proximity greatly reduces the number of candidate genes over using a $1 \mathrm{MB}$ window 681 (avg=18) or topologically associated domain (TAD) boundaries (avg=7). (B) T2D islet 682 enhancer signals formed chromatin loops with, or were in proximity to, an average of 2 683 target genes. (C) Target genes of T2D enhancer signals were strongly enriched for 684 biological processes related to protein secretion, protein transport, vesicles and vesicle 685 membranes, and endoplasmic reticulum (FDR<.2) (top), and target genes with islet 686 eQTL evidence were specifically enriched for vesicle-mediated transport $($ FDR $<2$ ) 687 (bottom). (D) Target genes with islet eQTLs to T2D islet enhancer signals (corrected 688 eQTL $P<.05$; $p$-values reported in table are uncorrected) and evidence that T2D and 689 eQTL signals are co-localized. (E) At the CDC123/CAMK1D locus T2D islet enhancer 690 variant rs11257655 was in a chromatin loop to the CAMK1D promoter and an islet eQTL 691 for CAMK1D expression. Probabilities (PPA) that variants are causal for T2D risk (top) 692 and variant association (-log10 P) with islet expression level of CAMK1D (middle).

694 Figure 5. Reduced IGF2BP2 activity in mouse islets impairs glucose-stimulated 695 insulin secretion during insulin resistance. (A) Immunostaining of insulin and IMP2 696 in mouse pancreas. (B) Expression of IMP2 in islets and other T2D-relevant tissues liver, 697 adipose, muscle, and brain. (C) Blood glucose, insulin, c-peptide and glucagon level 698 in 10-week-old male mice on high fat diet (HFD) ( $N=9$ ). Wild-type (black) and 699 Imp2ff/RIP2-Cre (red). (D) $1 \mathrm{~g} / \mathrm{kg}$ glucose was administered intraperitoneally after 700 overnight fasting of 12-week-old Imp2ff (black; N=10) and Imp2ff/RIP2-Cre (red; N=10) 
701 male mice maintained on normal chow diet (NCD). left=blood glucose; right=serum

702 insulin. (E) $1 \mathrm{~g} / \mathrm{kg}$ glucose was administered intraperitoneally after overnight fasting to

703 12-week-old Imp2ff (black; N=9) and Imp2ff/RIP2-Cre (red; N=9) male mice maintained

704 on NCD. left=blood glucose; right=serum insulin. Values represent mean and SD.

$705 \quad{ }^{*} \mathrm{P}<.05,{ }^{* *} \mathrm{P}<.01$

706

\section{Supplemental Figures:}

Figure S1. Characteristics of pancreatic islet regulatory elements (A) Heatmap of the Pearson correlation of ATAC seq signal across four islet samples, calculated as the

712 probabilities for the 9-state islet model from chromHMM, with individual ChIP-seq assays

713 shown on the $x$-axis and labelled chromatin states on the $y$-axis. (C) Heatmap showing

714 percentage of each class of islet regulatory elements mapping in 200bp bins around

715 GENCODE transcriptional start sites. (D) Percentage of ENCODE cell-types in DHS

716 sites overlapping each class of islet regulatory elements. (E) Jaccard overlap of each

717 class of islet regulatory elements with islet ChIP-seq sites for five transcription factors.

719 Figure S2. Characteristics of pancreatic islet chromatin loops (A) Heatmap showing

720 the Pearson correlation of Hi-C contacts across islet samples in $100 \mathrm{~kb}$ bins across the

721 genome. (B) Histogram of the distance between loop anchors in islets. (C) Histogram

722 of number of loops within $25 \mathrm{~kb}$ of each islet active enhancer to gene promoter regions.

723 (D) Boxplot showing genes with increasing numbers of chromatin loops to islet

724 enhancers (x-axis) had on average higher relative expression level in islets (y-axis). (E)

725 The number of chromatin loops to islet enhancers was a significant predictor of relative

726 gene expression level in islets but not 53 other tissues in GTEx. ${ }^{* *} P<.001$. Values

727 represent effect size and SE.

729 Figure S3. Effects of variants in pancreatic islet regulatory elements on T2D risk

730 (A) Enrichment Z-score measured using LD-score regression for each class of islet

731 regulatory elements (y-axis), subset by states that were (dark) or were not (light) within

732 25kb of a Hi-C loop anchor. (B) Enrichments from the fgwas genome-wide joint model

733 including islet active enhancers (EnhA1), flanking promoters (TssFlnk), and coding

734 exons (CDS). Values represent log enrichment and 95\% Cl. (C) Posterior causal 
735 probabilities (PPA) of variants within islet active enhancers in $1 \mathrm{MB}$ windows genome-

736 wide excluding known T2D risk loci. Blue $=$ variants with PPA $>0.01$, grey $=$ variants with

$737 \quad \mathrm{PPA}<0.01$.

738

739 Figure S4. T2D enhancer signal chromatin loops to candidate target genes. Re-

740 weighted posterior causal probabilities of variants (top), islet $\mathrm{Hi}-\mathrm{C}$ loops, chromatin

741 states and ATAC-seq signal (middle), and known genes (bottom), for (A) five

742 independent T2D risk signals at the KCNQ1 locus, (B) T2D signal at the ZMIZ1 locus,

743 and (C) T2D signal at the KCNJ11/ABCC8 locus. For (A), posterior probabilities are

744 shown in different colors for each of the five independent signals.

745

746 Figure S5. Characterization of mice after conditional IGF2BP2 ablation in beta

747 cells. (A) Schematic representation of the wild type Imp2 allele showing exon 1-2 and

748 flanking intron sequences, and the $\operatorname{lmp} 2^{\text {flox }}$ targeted allele. (B) Body weight for wild-type,

749 RIP2-Cre, Imp2 $2^{f f}$ and Imp2 $2^{\text {ff }} /$ RIP2-Cre mice on normal chow diet (top) and high fat diet

750 (bottom). (C) Insulin tolerance tests in 14-week-old Imp2 $2^{\mathrm{ff}} / \mathrm{RIP2}$-Cre (red) and Imp2 $2^{\mathrm{ff}}$

751 (black) mice on a normal chow diet (left) and high fat diet (right). Values represent mean

752 and SD.

753

754

755

Supplemental Tables:

756 STable 1: Donor and sequencing characteristics of pancreatic islet samples

757 STable 2: Regulatory elements in pancreatic islets

758 STable 3: Sequence motifs enriched in islet regulatory elements

759 STable 4: Hi-C loops identified in pancreatic islet samples

760 STable 5: Target gene chromatin loops of islet enhancer elements

761 STable 6: Islet enhancer chromatin loops of gene promoter regions

762 STable 7: Functional annotations enriched in genes with multiple enhancer interactions

763 STable 8: T2D candidate causal variants in islet active enhancers

764 STable 9: Target genes of T2D islet enhancer signals

765 STable 10: Gene set annotations enriched in target genes of T2D islet enhancer signals

766 STable 11: Target genes with islet eQTLs for T2D islet enhancer signals 
7701 Fuchsberger, C. et al. The genetic architecture of type 2 diabetes. Nature 536,

771

772

773

774

775

776

777

778

779

780

781

782

783

784

785

786

787

788

789

790

791

792

793

794

795

796

797

798

799

800

801

802

803

804

805

806

807

808

809

810

811

812

813

814

815

816

817

818

819
41-47, doi:10.1038/nature18642 (2016).

2 Gaulton, K. J. et al. Genetic fine mapping and genomic annotation defines causal mechanisms at type 2 diabetes susceptibility loci. Nat Genet 47, 1415-1425, doi:10.1038/ng.3437 (2015).

3 Gaulton, K. J. Mechanisms of Type 2 Diabetes Risk Loci. Curr Diab Rep 17, 72, doi:10.1007/s11892-017-0908-x (2017).

4 Pasquali, L. et al. Pancreatic islet enhancer clusters enriched in type 2 diabetes risk-associated variants. Nat Genet 46, 136-143, doi:10.1038/ng.2870 (2014).

5 Parker, S. C. et al. Chromatin stretch enhancer states drive cell-specific gene regulation and harbor human disease risk variants. Proc Natl Acad Sci U S A 110, 17921-17926, doi:10.1073/pnas.1317023110 (2013).

6 Stitzel, M. L. et al. Global epigenomic analysis of primary human pancreatic islets provides insights into type 2 diabetes susceptibility loci. Cell Metab 12, 443-455, doi:10.1016/j.cmet.2010.09.012 (2010).

7 Varshney, A. et al. Genetic regulatory signatures underlying islet gene expression and type 2 diabetes. Proc Natl Acad Sci U S A 114, 2301-2306, doi:10.1073/pnas.1621192114 (2017).

8 Rao, S. S. et al. A 3D map of the human genome at kilobase resolution reveals principles of chromatin looping. Cell 159, 1665-1680, doi:10.1016/j.cell.2014.11.021 (2014).

9 Won, H. et al. Chromosome conformation elucidates regulatory relationships in developing human brain. Nature 538, 523-527, doi:10.1038/nature19847 (2016).

10 Dixon, J. R. et al. Topological domains in mammalian genomes identified by analysis of chromatin interactions. Nature 485, 376-380, doi:10.1038/nature11082 (2012).

11 Buenrostro, J. D., Giresi, P. G., Zaba, L. C., Chang, H. Y. \& Greenleaf, W. J. Transposition of native chromatin for fast and sensitive epigenomic profiling of open chromatin, DNA-binding proteins and nucleosome position. Nat Methods 10, 1213-1218, doi:10.1038/nmeth.2688 (2013).

12 Zhang, Y. et al. Model-based analysis of ChIP-Seq (MACS). Genome Biol 9, R137, doi:10.1186/gb-2008-9-9-r137 (2008).

13 Pasquali, L. et al. Pancreatic islet enhancer clusters enriched in type 2 diabetes risk-associated variants. Nature genetics 46, 136-143, doi:10.1038/ng.2870 (2014).

14 Ernst, J. \& Kellis, M. ChromHMM: automating chromatin-state discovery and characterization. Nat Methods 9, 215-216, doi:10.1038/nmeth.1906 (2012).

15 Gaulton, K. J. et al. A map of open chromatin in human pancreatic islets. Nat Genet 42, 255-259, doi:10.1038/ng.530 (2010).

$16 \mathrm{Yu}, \mathrm{M}$. \& Ren, B. The Three-Dimensional Organization of Mammalian Genomes. Annu Rev Cell Dev Biol 33, 265-289, doi:10.1146/annurev-cellbio-100616060531 (2017).

17 Yan, J. et al. Histone $\mathrm{H} 3$ lysine 4 monomethylation modulates long-range chromatin interactions at enhancers. Cell Res, doi:10.1038/cr.2018.1 (2018).

18 van de Bunt, M. et al. Transcript Expression Data from Human Islets Links Regulatory Signals from Genome-Wide Association Studies for Type 2 Diabetes and Glycemic Traits to Their Downstream Effectors. PLoS Genet 11, e1005694, doi:10.1371/journal.pgen.1005694 (2015).

19 Consortium, G. T. et al. Genetic effects on gene expression across human tissues. Nature 550, 204-213, doi:10.1038/nature24277 (2017). 
82020 Pickrell, J. K. Joint analysis of functional genomic data and genome-wide

821

822

823

824

825

826

827

828

829

830

831

832

833

834

835

836

837

838

839

840

841

842

843

844

845

846

847

848

849

850

851

852

853

854

855

856

857

858

859

860

861

862

863

864

865

866

867

868

869 association studies of 18 human traits. Am J Hum Genet 94, 559-573, doi:10.1016/j.ajhg.2014.03.004 (2014).

21 Scott, R. A. et al. An Expanded Genome-Wide Association Study of Type 2 Diabetes in Europeans. Diabetes 66, 2888-2902, doi:10.2337/db16-1253 (2017).

22 Wood, A. R. et al. A Genome-Wide Association Study of IVGTT-Based Measures of First-Phase Insulin Secretion Refines the Underlying Physiology of Type 2 Diabetes Variants. Diabetes 66, 2296-2309, doi:10.2337/db16-1452 (2017).

23 Fogarty, M. P., Cannon, M. E., Vadlamudi, S., Gaulton, K. J. \& Mohlke, K. L. Identification of a regulatory variant that binds FOXA1 and FOXA2 at the CDC123/CAMK1D type 2 diabetes GWAS locus. PLoS Genet 10, e1004633, doi:10.1371/journal.pgen.1004633 (2014).

24 Dai, N. et al. mTOR phosphorylates IMP2 to promote IGF2 mRNA translation by internal ribosomal entry. Genes Dev 25, 1159-1172, doi:10.1101/gad.2042311 (2011).

$25 \mathrm{Li}, \mathrm{H}$. \& Durbin, R. Fast and accurate short read alignment with Burrows-Wheeler transform. Bioinformatics 25, 1754-1760, doi:10.1093/bioinformatics/btp324 (2009).

26 Consortium, E. P. An integrated encyclopedia of DNA elements in the human genome. Nature 489, 57-74, doi:10.1038/nature11247 (2012).

$27 \mathrm{Li}, \mathrm{H}$. Aligning sequence reads, clone sequences and assembly contigs with BWA-MEM. ArXiv e-prints $1303 \quad$ (2013). <http://adsabs.harvard.edu/abs/2013arXiv1303.3997L>.

28 Durand, N. C. et al. Juicebox Provides a Visualization System for Hi-C Contact Maps with Unlimited Zoom. Cell Syst 3, 99-101, doi:10.1016/j.cels.2015.07.012 (2016).

29 Greenwald, W. W. et al. Pgltools: a genomic arithmetic tool suite for manipulation of Hi-C peak and other chromatin interaction data. BMC Bioinformatics 18, 207, doi:10.1186/s12859-017-1621-0 (2017).

$30 \mathrm{Li}, \mathrm{H}$. \& Durbin, R. Fast and accurate long-read alignment with Burrows-Wheeler transform. Bioinformatics 26, 589-595, doi:10.1093/bioinformatics/btp698 (2010).

31 Heinz, S. et al. Simple combinations of lineage-determining transcription factors prime cis-regulatory elements required for macrophage and B cell identities. Mol Cell 38, 576-589, doi:10.1016/j.molcel.2010.05.004 (2010).

32 Harrow, J. et al. GENCODE: the reference human genome annotation for The ENCODE Project. Genome Res 22, 1760-1774, doi:10.1101/gr.135350.111 (2012).

33 Subramanian, A. et al. Gene set enrichment analysis: a knowledge-based approach for interpreting genome-wide expression profiles. Proc Natl Acad Sci U S A 102, 15545-15550, doi:10.1073/pnas.0506580102 (2005).

34 Wakefield, J. A Bayesian measure of the probability of false discovery in genetic epidemiology studies. Am J Hum Genet 81, 208-227, doi:10.1086/519024 (2007).

35 Quinlan, A. R. \& Hall, I. M. BEDTools: a flexible suite of utilities for comparing genomic features. Bioinformatics 26, 841-842, doi:10.1093/bioinformatics/btq033 (2010).

36 Bailey, T. L. DREME: motif discovery in transcription factor ChIP-seq data. Bioinformatics 27, 1653-1659, doi:10.1093/bioinformatics/btr261 (2011).

37 Bailey, T. L., Johnson, J., Grant, C. E. \& Noble, W. S. The MEME Suite. Nucleic Acids Res 43, W39-49, doi:10.1093/nar/gkv416 (2015). 
87038 Bryne, J. C. et al. JASPAR, the open access database of transcription factor-

871

872

873

874

875

876

877

878

879 binding profiles: new content and tools in the 2008 update. Nucleic Acids Res $\mathbf{3 6}$, D102-106, doi:10.1093/nar/gkm955 (2008).

39 Jolma, A. et al. Multiplexed massively parallel SELEX for characterization of human transcription factor binding specificities. Genome Res 20, 861-873, doi:10.1101/gr.100552.109 (2010).

40 Li, B., Ruotti, V., Stewart, R. M., Thomson, J. A. \& Dewey, C. N. RNA-Seq gene expression estimation with read mapping uncertainty. Bioinformatics 26, 493500, doi:10.1093/bioinformatics/btp692 (2010).

884

885

41 Fadista, J. et al. Global genomic and transcriptomic analysis of human pancreatic islets reveals novel genes influencing glucose metabolism. Proc Natl Acad Sci U $S$ A 111, 13924-13929, doi:10.1073/pnas.1402665111 (2014).

42 Willer, C. J., Li, Y. \& Abecasis, G. R. METAL: fast and efficient meta-analysis of genomewide association scans. Bioinformatics 26, 2190-2191, doi:10.1093/bioinformatics/btq340 (2010).

886

43 Giambartolomei, C. et al. Bayesian test for colocalisation between pairs of genetic association studies using summary statistics. PLoS Genet 10, e1004383, doi:10.1371/journal.pgen.1004383 (2014). 


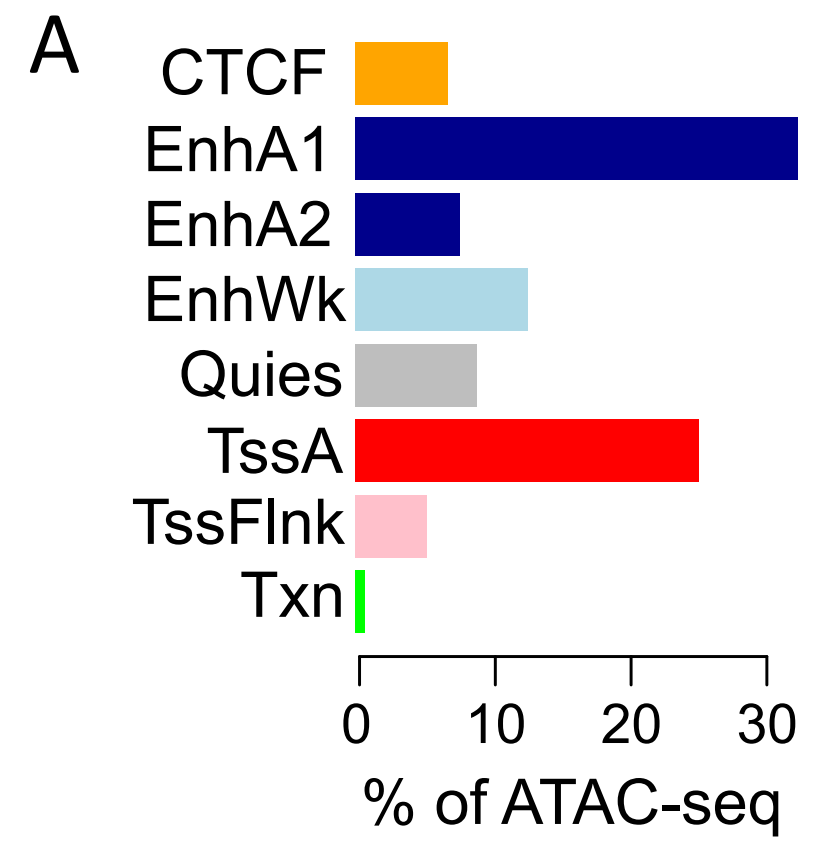

C

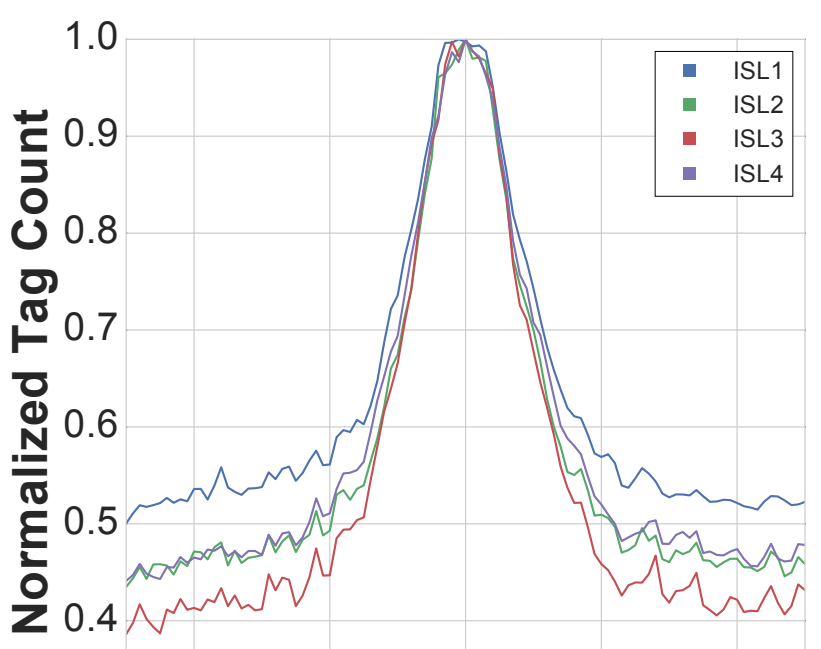
0.3
$\begin{array}{lllll}\circ & \circ & \circ & \circ & \circ \\ & \circ & \circ & \circ \\ \text { ㄱ } & & & \circ\end{array}$
Distance (kb) from loop anchor midpoint

D

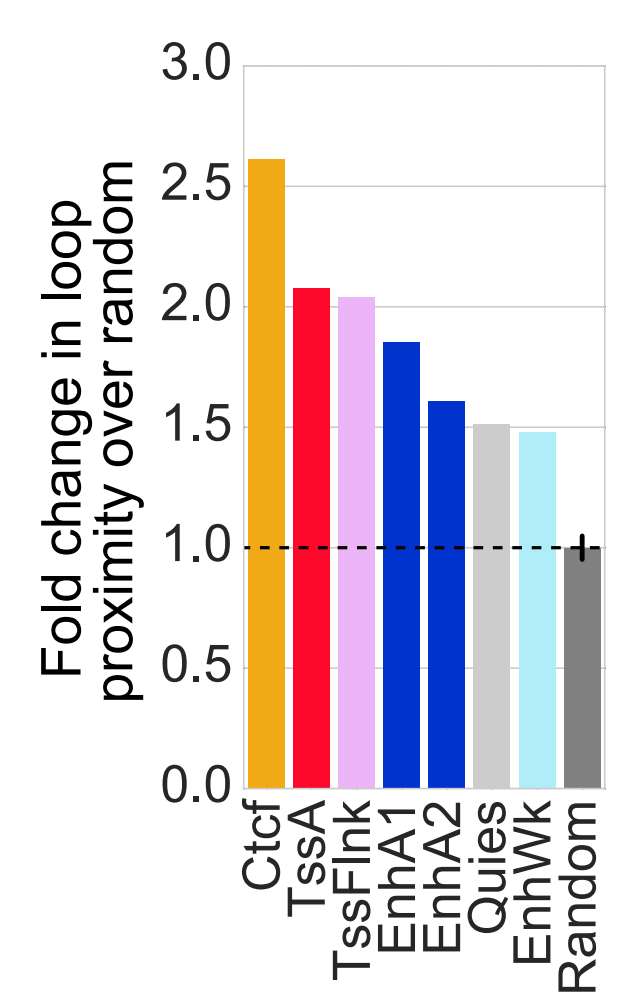

B

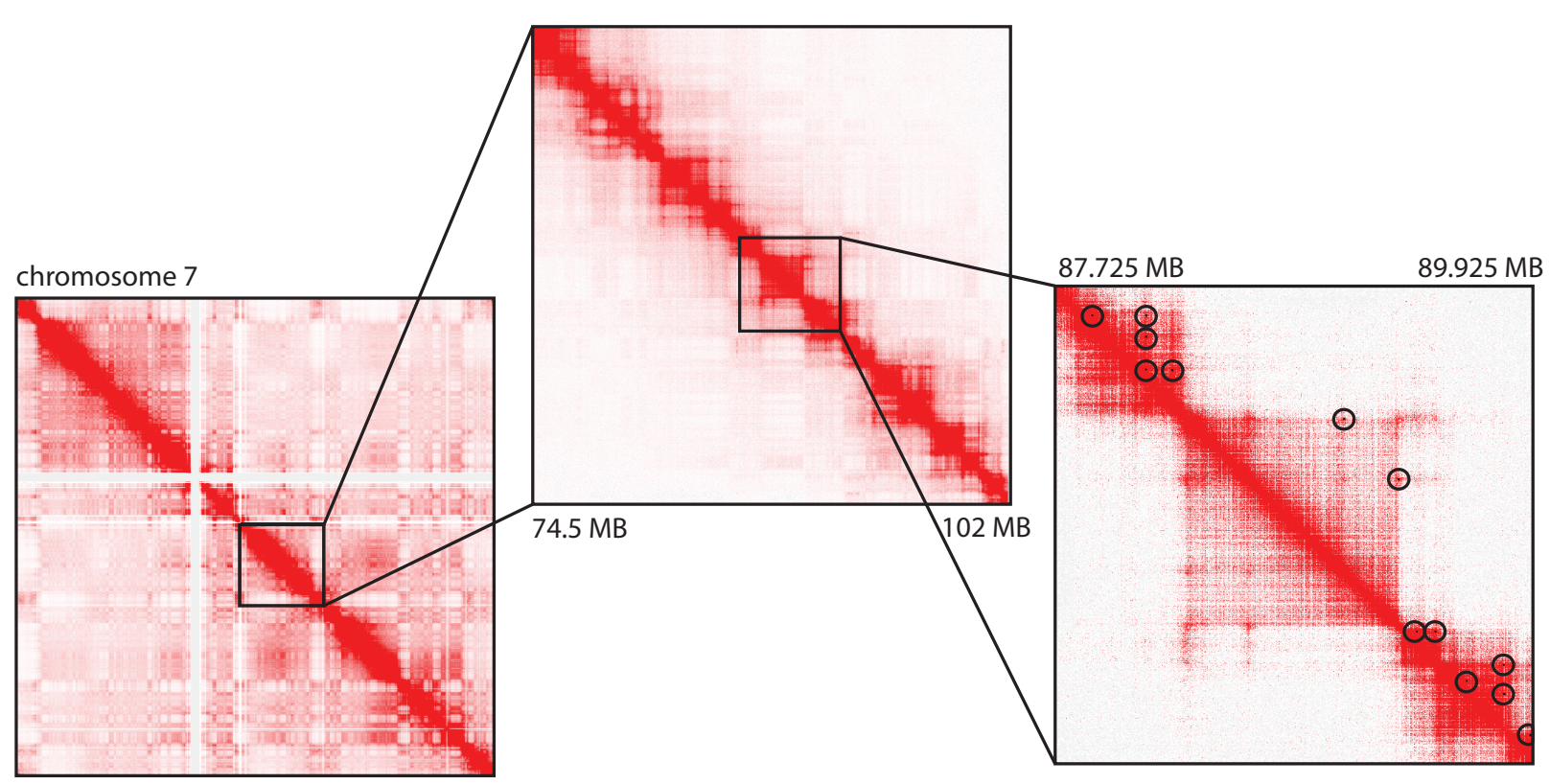

$E$

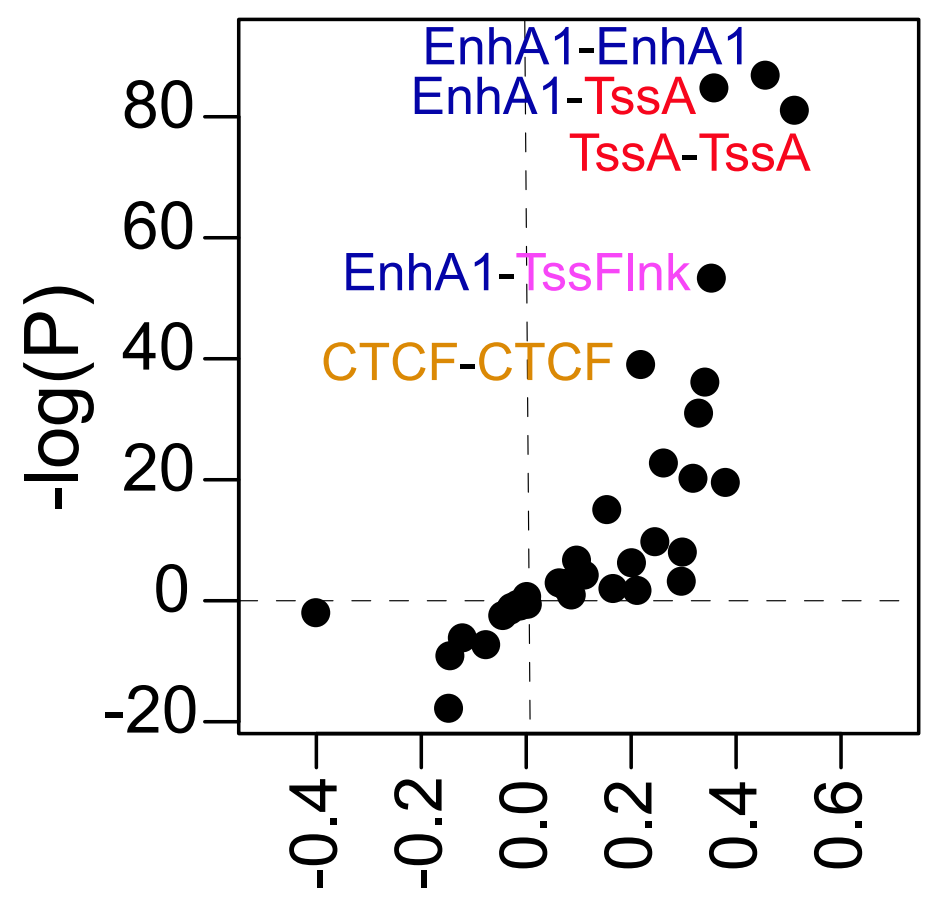

$\mathrm{Hi}-\mathrm{C}$ interaction ratio

Figure 1. Chromatin accessibility and conformation in pancreatic islets 
A

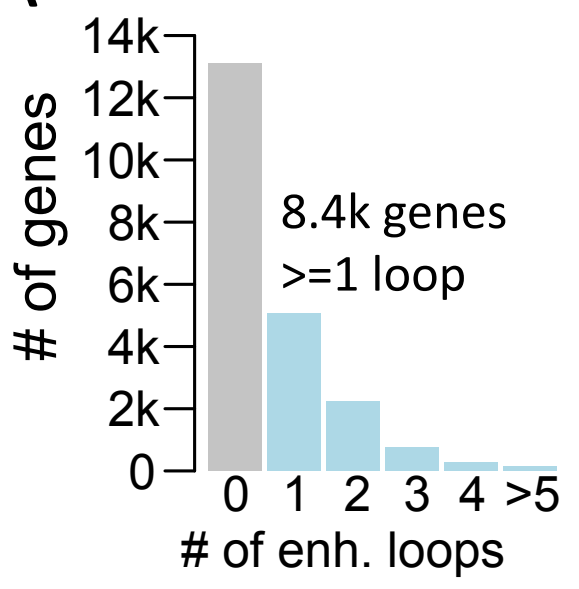

C

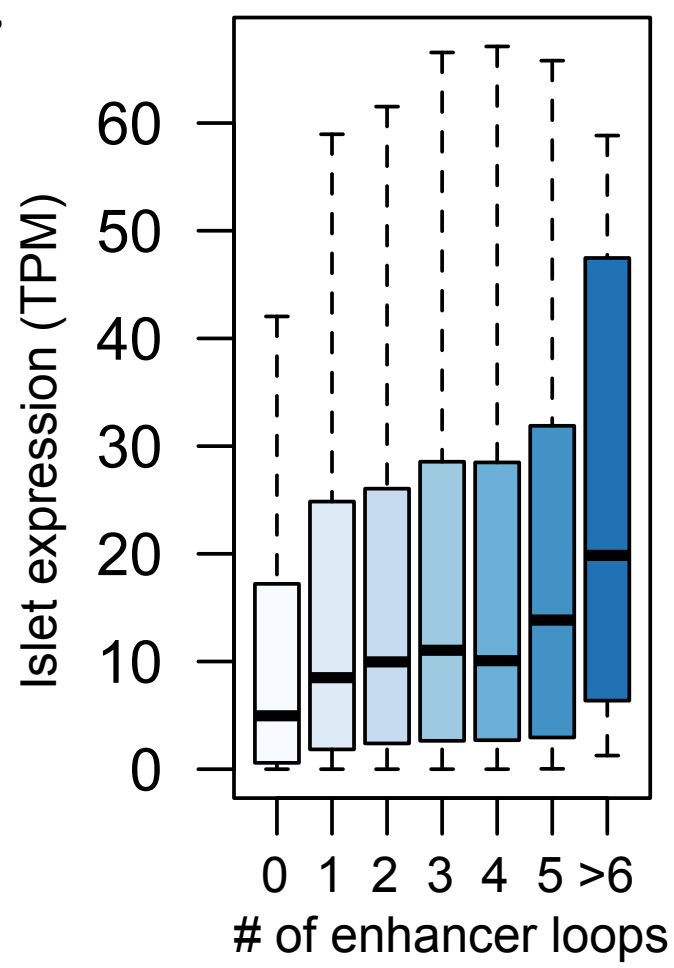

$38,000,000$

$1 \mathrm{MB}$
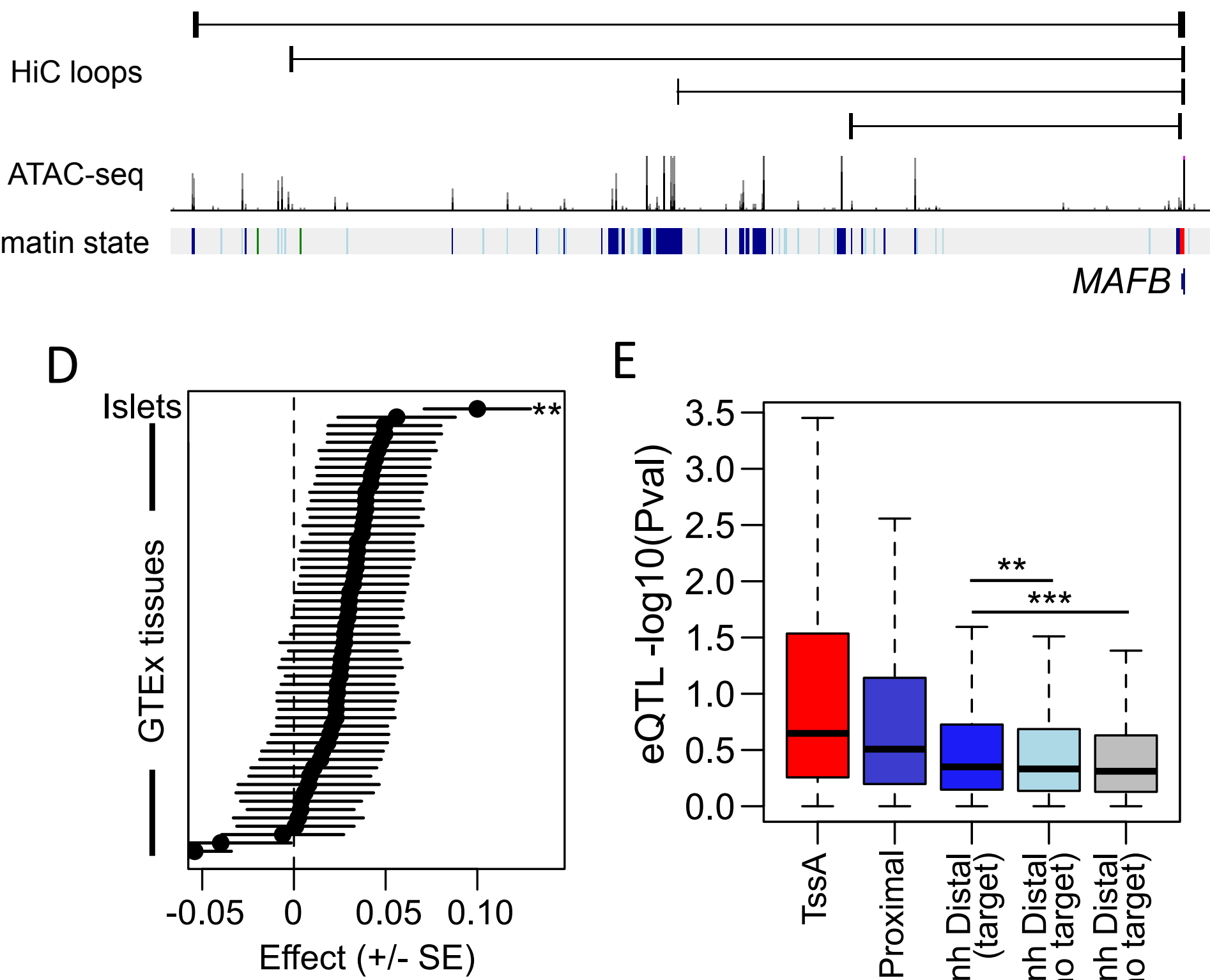

$E$

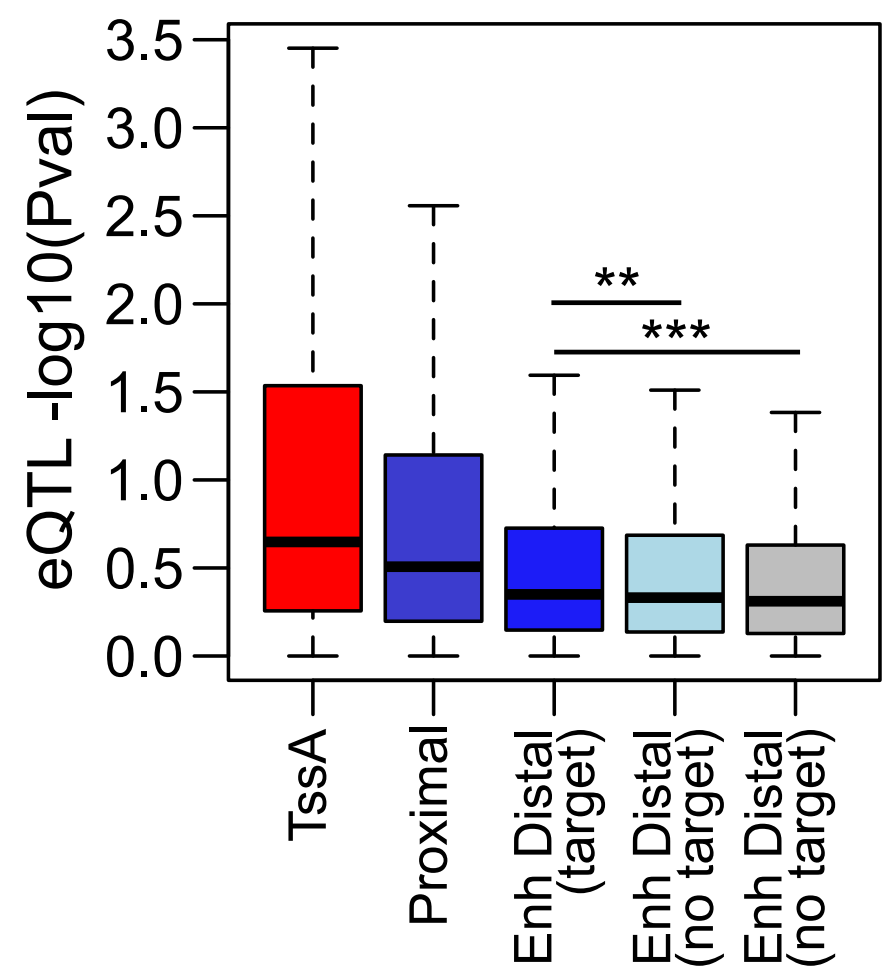

Figure 2. Enhancer regulation of target gene expression in islets 
A

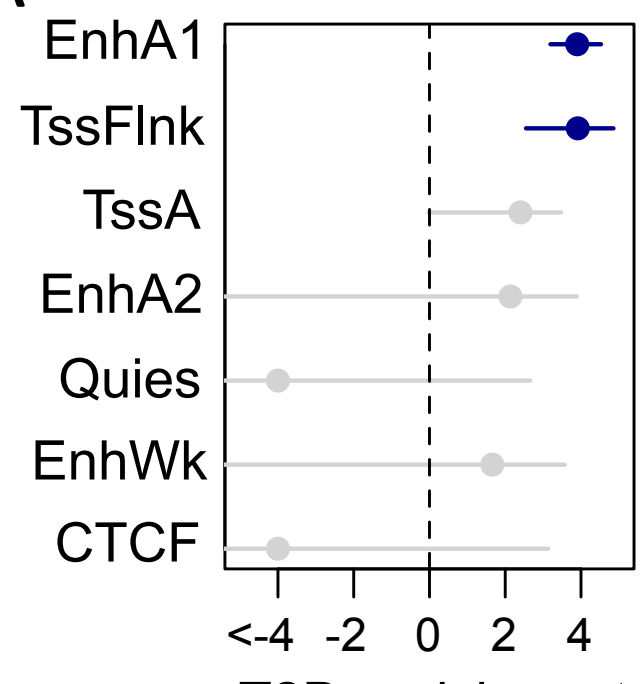

T2D enrichment
B

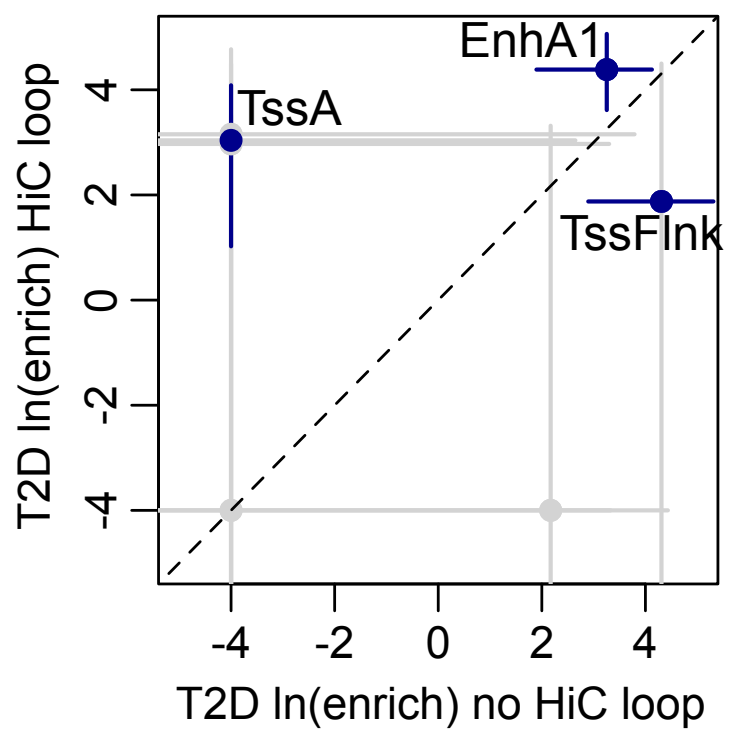

C

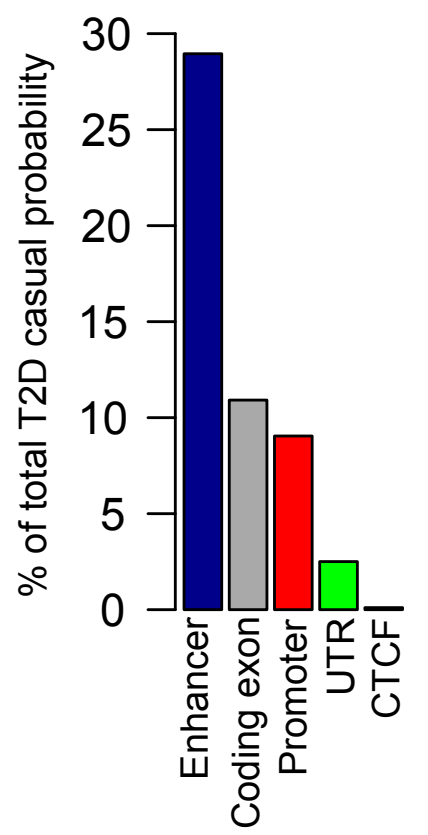

D

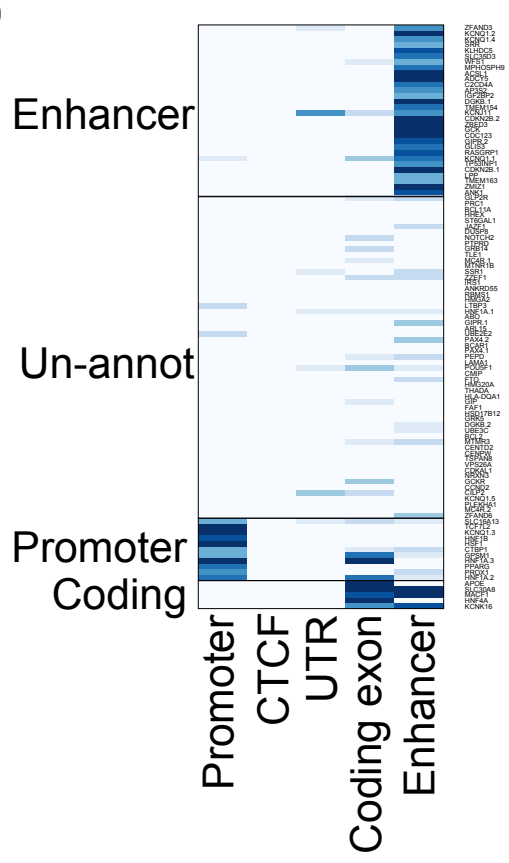

$\mathrm{H}$

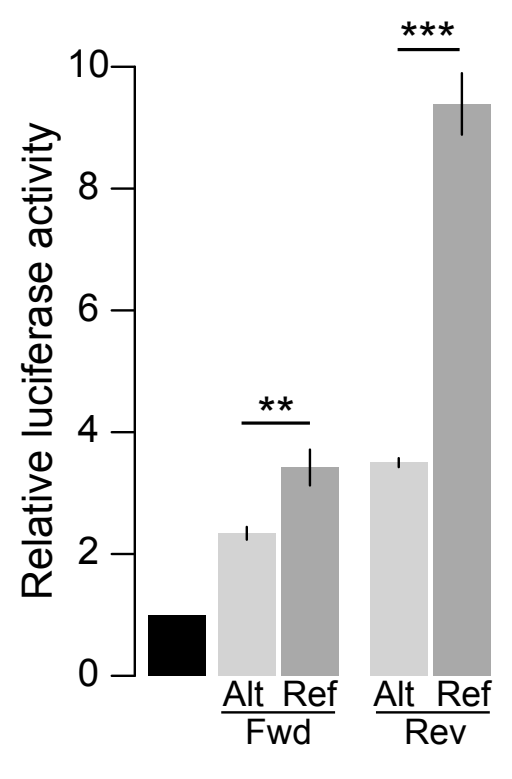

Figure 3. Type 2 diabetes risk signals map in islet enhancers 
A

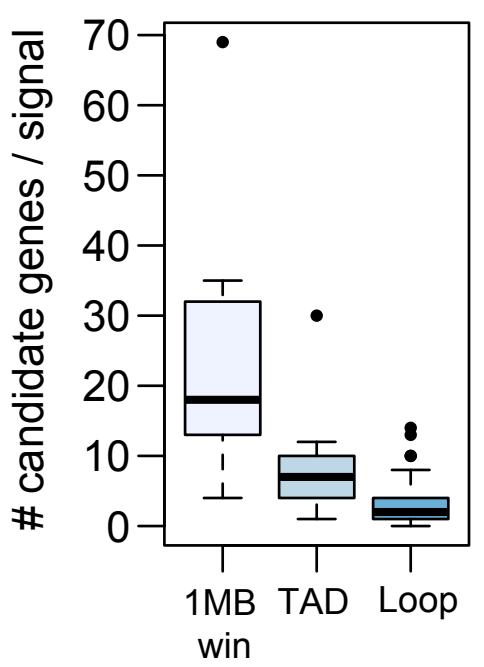

B

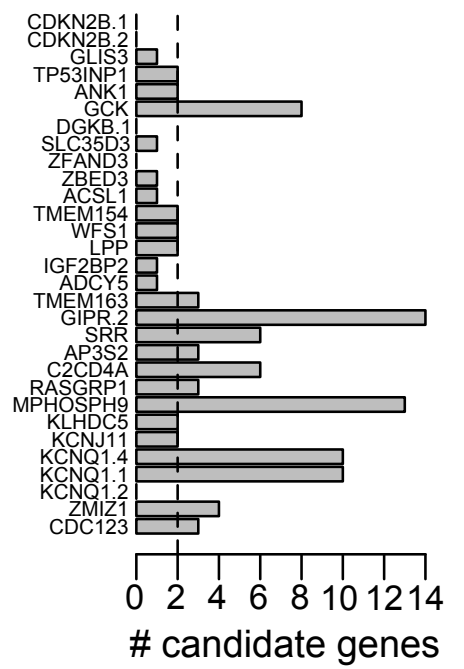

D

\begin{tabular}{llllrr}
\hline Variant & $\begin{array}{l}\text { Locus } \\
\text { name }\end{array}$ & $\begin{array}{l}\text { \# target } \\
\text { genes }\end{array}$ & eQTL gene & $\begin{array}{r}\text { eQTL p- } \\
\text { value }\end{array}$ & $\begin{array}{r}\text { Shared } \\
\text { eQTL }\end{array}$ \\
\hline rs11257655 & CDC123 & 3 & CAMK1D & $1.72 \mathrm{E}-14$ & $\mathrm{Y}$ \\
rs508419 & ANK1 & 2 & NKX6-3 & $5.59 \mathrm{E}-10$ & $\mathrm{Y}$ \\
rs1260294 & MPHOSPH9 13 & ABCB9 & $2.63 \mathrm{E}-07$ & $\mathrm{Y}$ \\
rs7646518 & IGF2BP2 & 1 & IGF2BP2 & $7.49 \mathrm{E}-07$ & $\mathrm{Y}$ \\
rs4954179 & TMEM163 & 3 & ACMSD & $5.43 \mathrm{E}-06$ & $\mathrm{Y}$ \\
rs4954179 & TMEM163 & 3 & TMEM163 & $9.69 \mathrm{E}-05$ & $\mathrm{Y}$ \\
rs17205526 & C2CD4A/B & 6 & C2CD4B & 0.00088 & $\mathrm{Y}$ \\
rs116401167 & ACSL1 & 1 & ACSL1 & 0.040 & $\mathrm{Y}$ \\
rs7732130 & ZBED3 & 1 & PDE8B & 0.048 & $\mathrm{Y}$ \\
\hline
\end{tabular}

$\mathrm{E}$

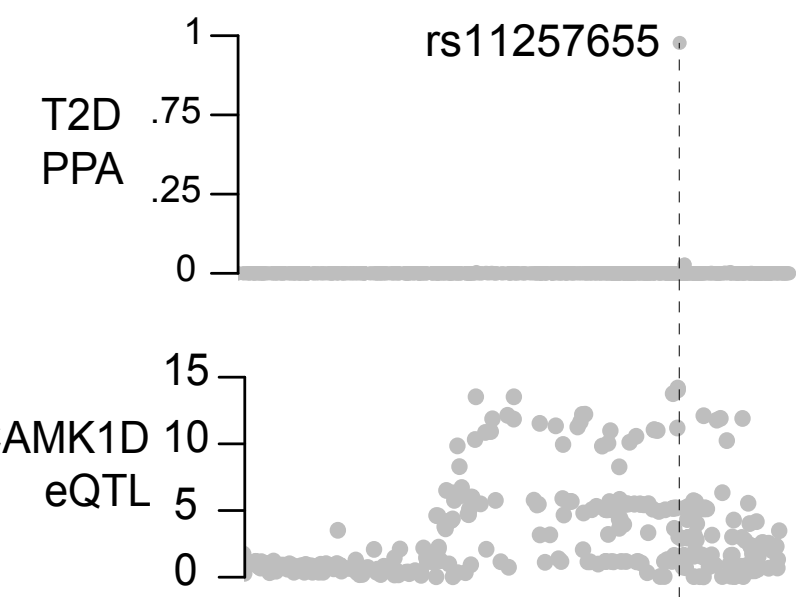

$\mathrm{HiC}$ loops

Chromatin state

ATAC-seq

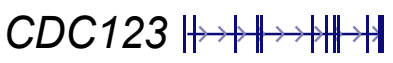

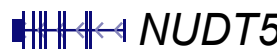

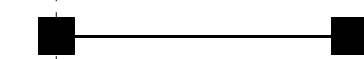

I II

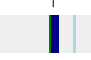

CAMK1D - $\log 10($ P-value)

Figure 4. Target genes of type 2 diabetes islet enhancer signals 

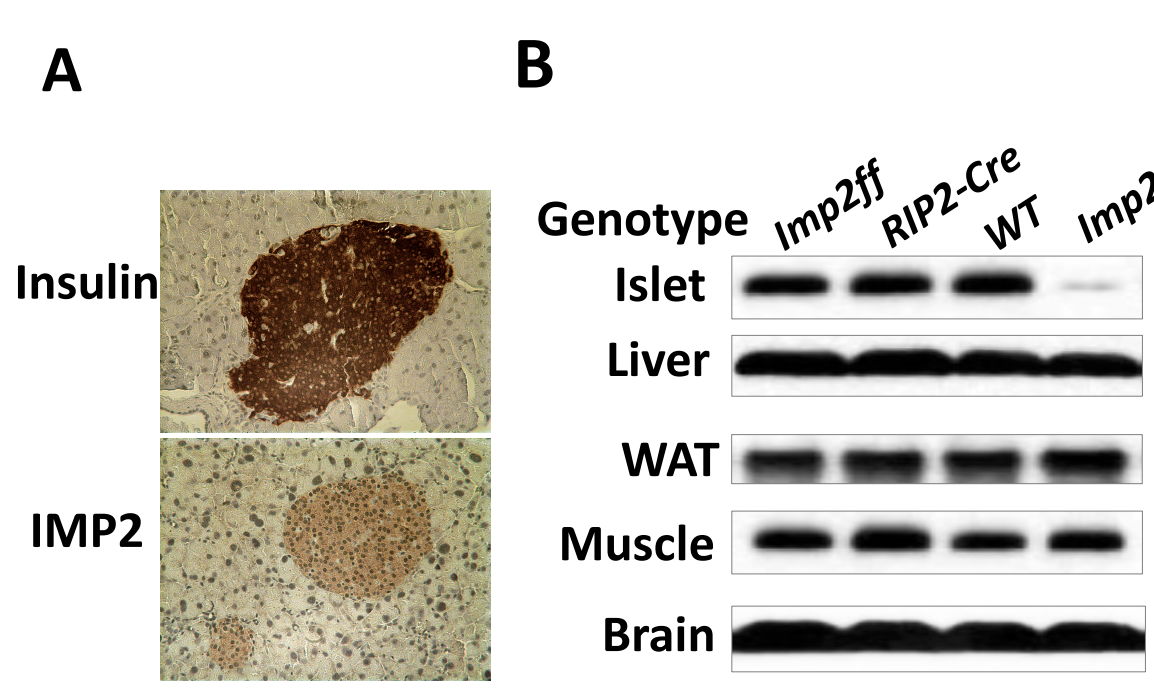

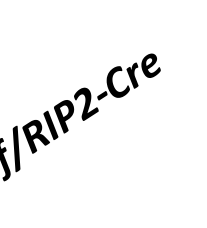

C $-w T$

- Imp2ff/RIP2-Cre
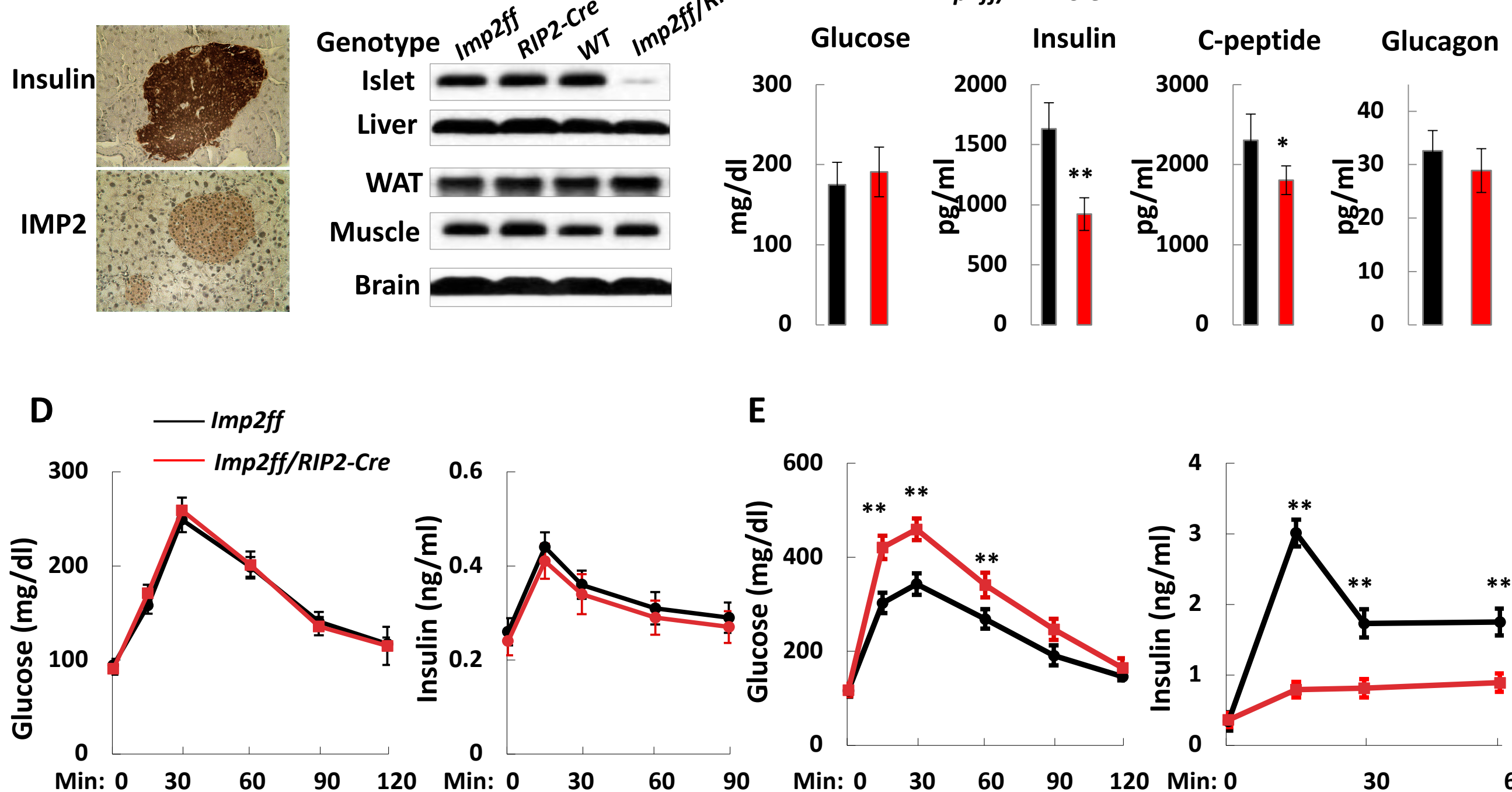

Figure 5. Reduced IGF2BP2 activity in mouse islets impairs glucose-stimulated insulin secretion in diet-induced insulin resistance 

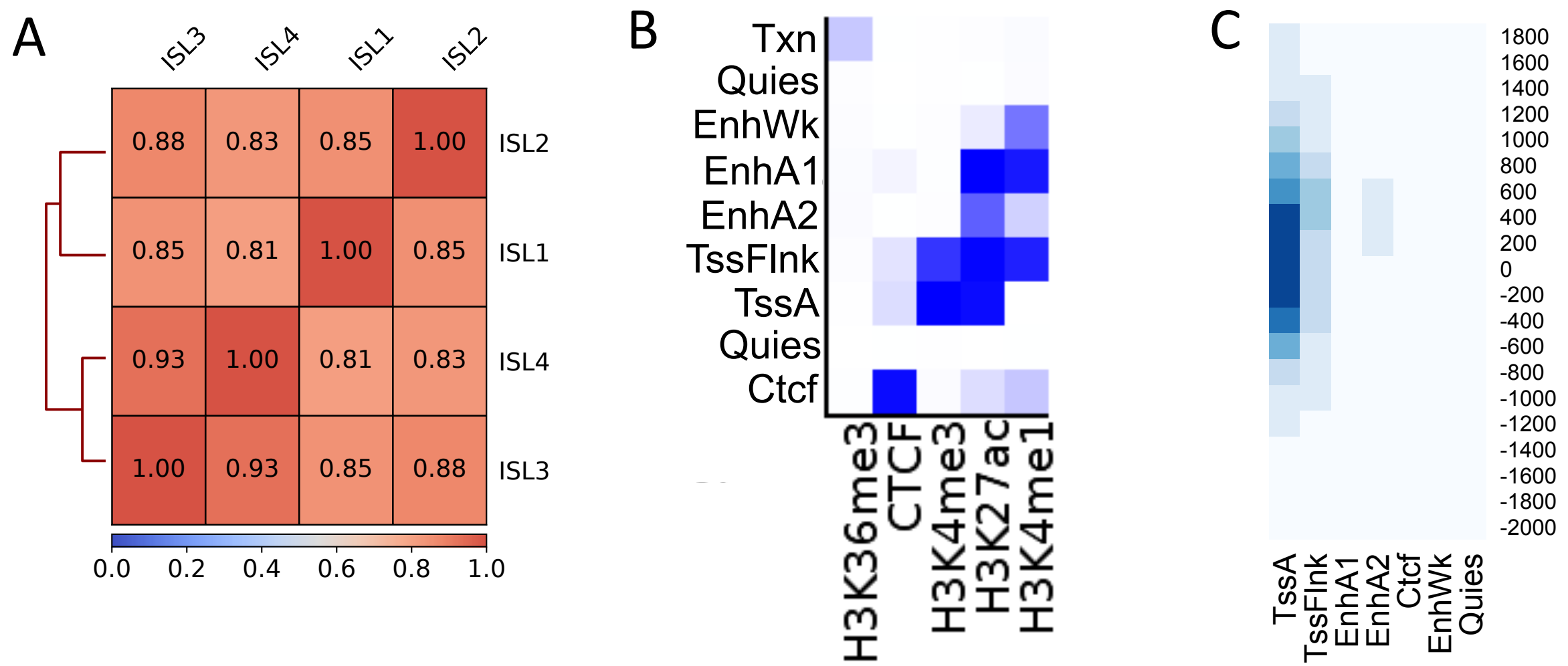

D

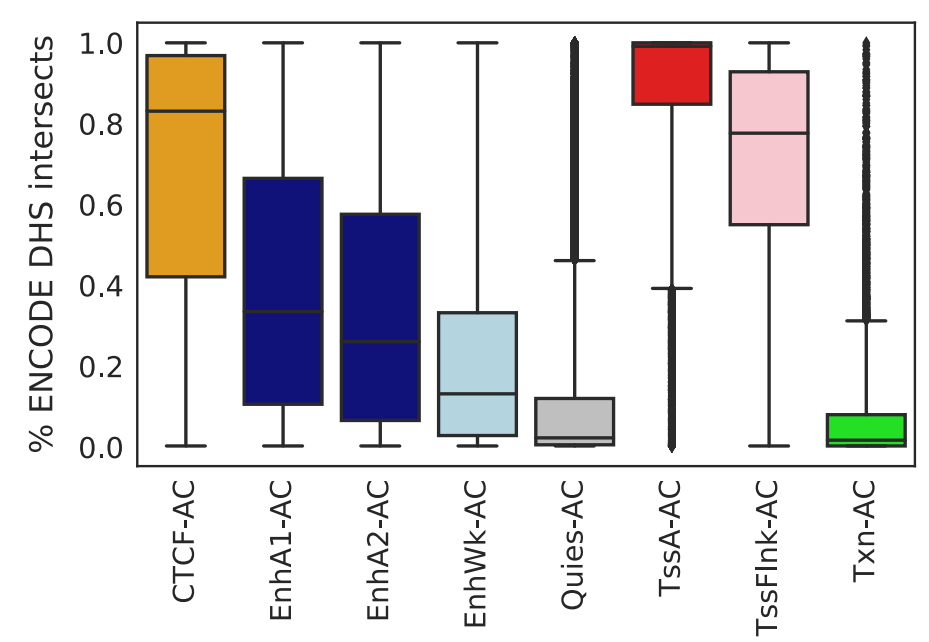

$E$

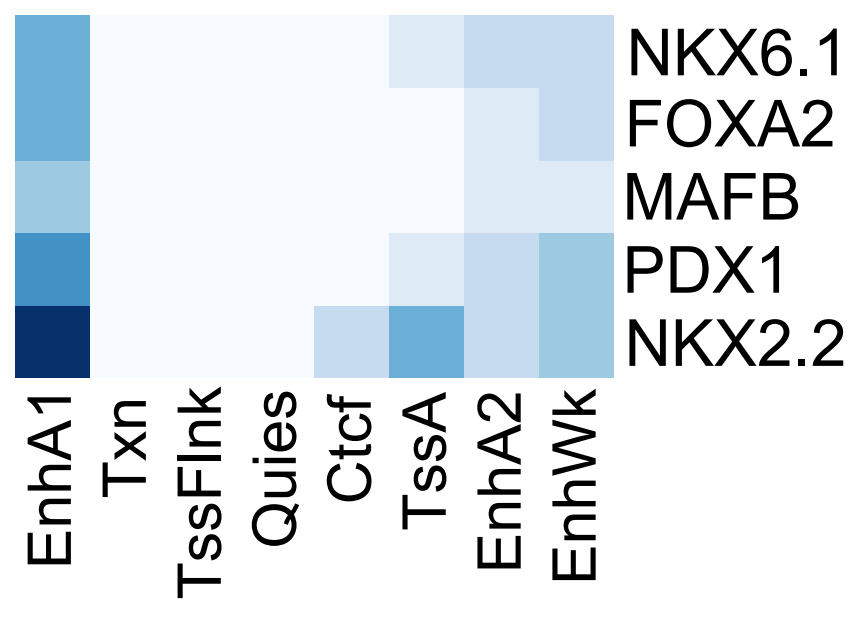

Figure S1. Characteristics of pancreatic islet regulatory elements 
A

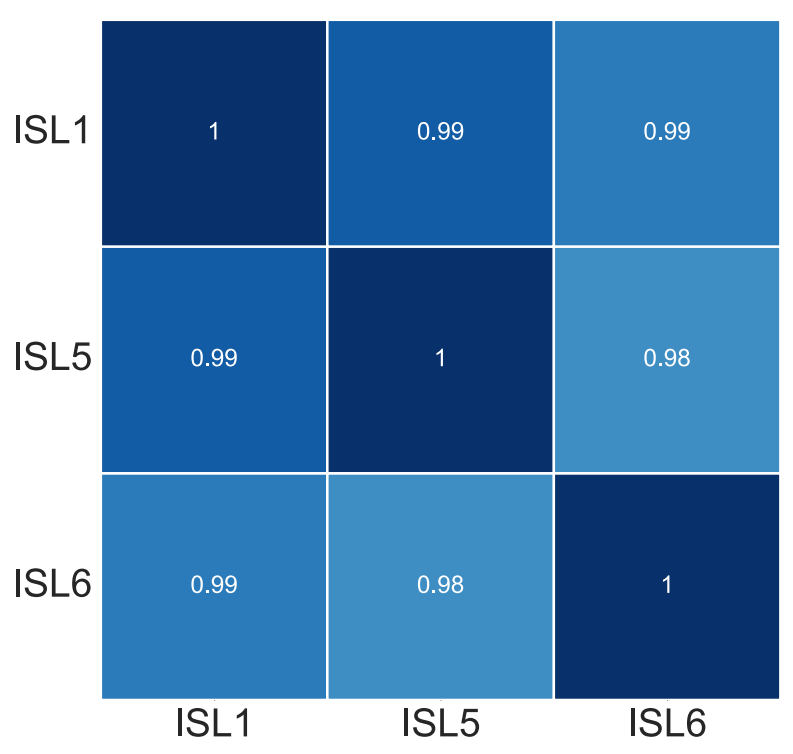

C

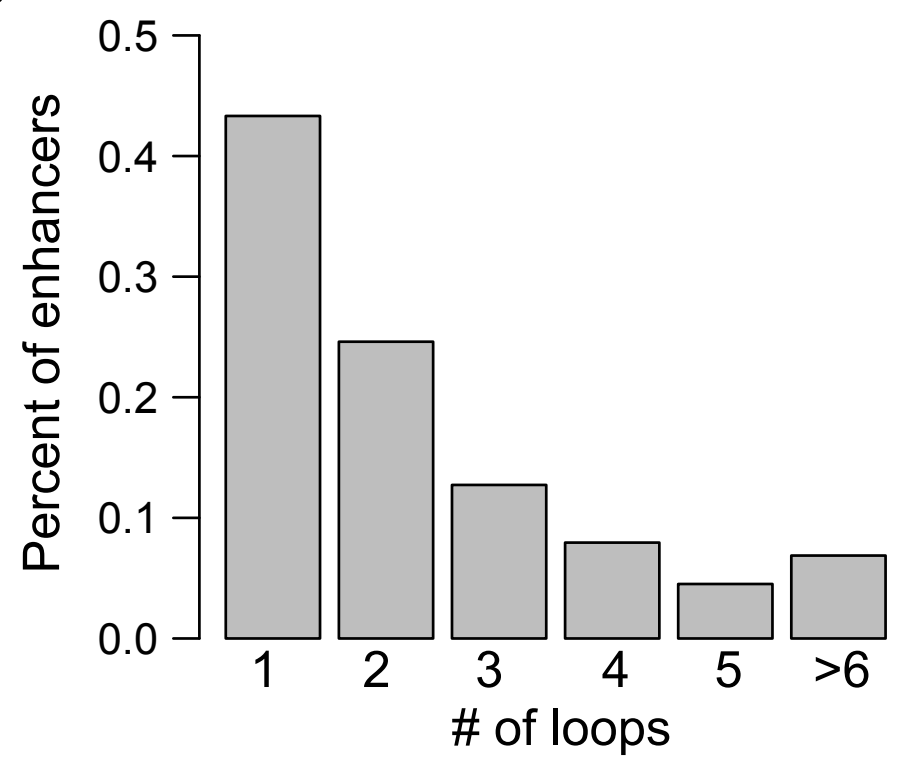

B

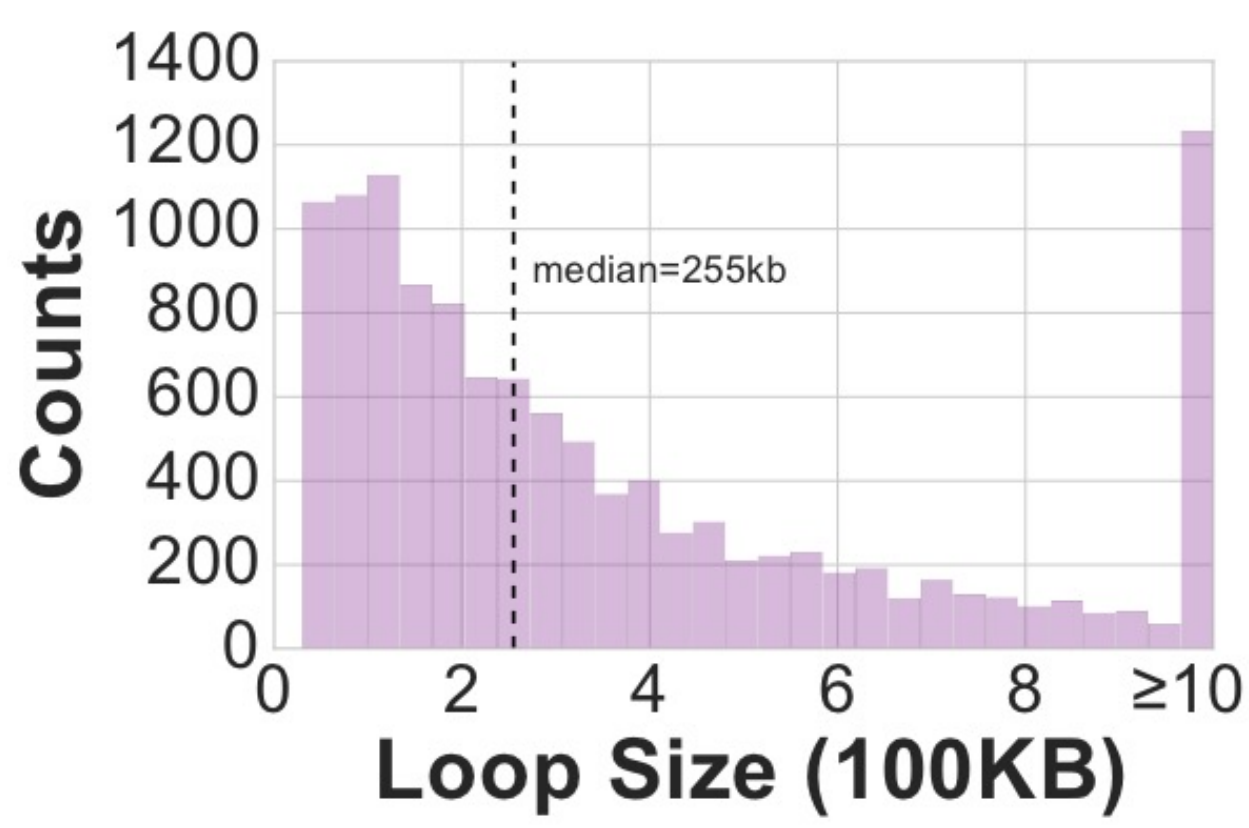

D

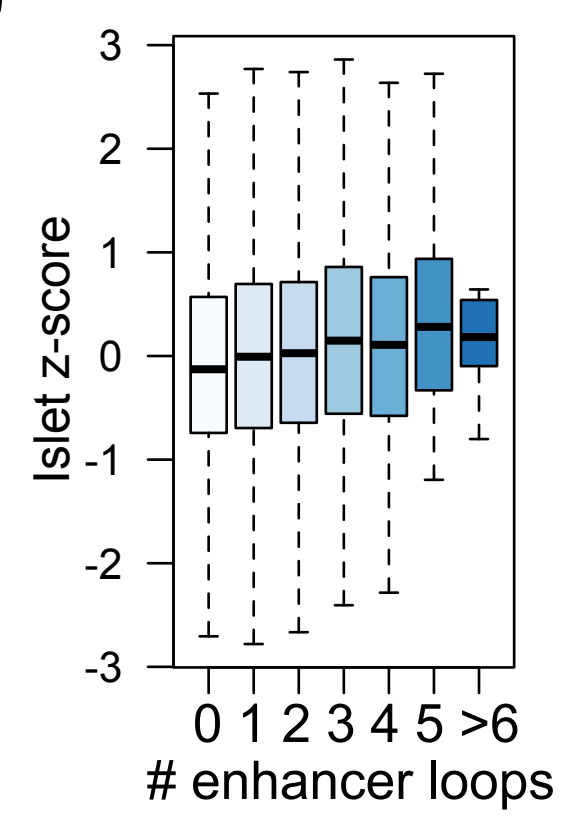

E

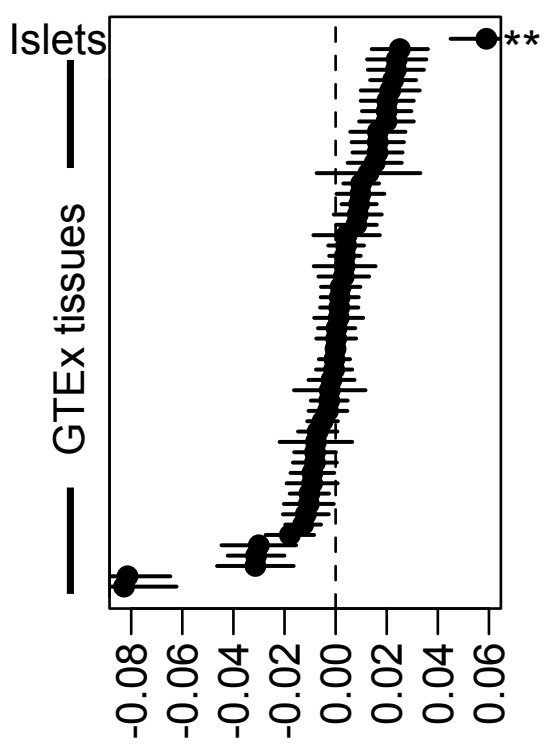

Effect (+/- SE)

Figure S2. Characteristics of pancreatic islet chromatin loops 

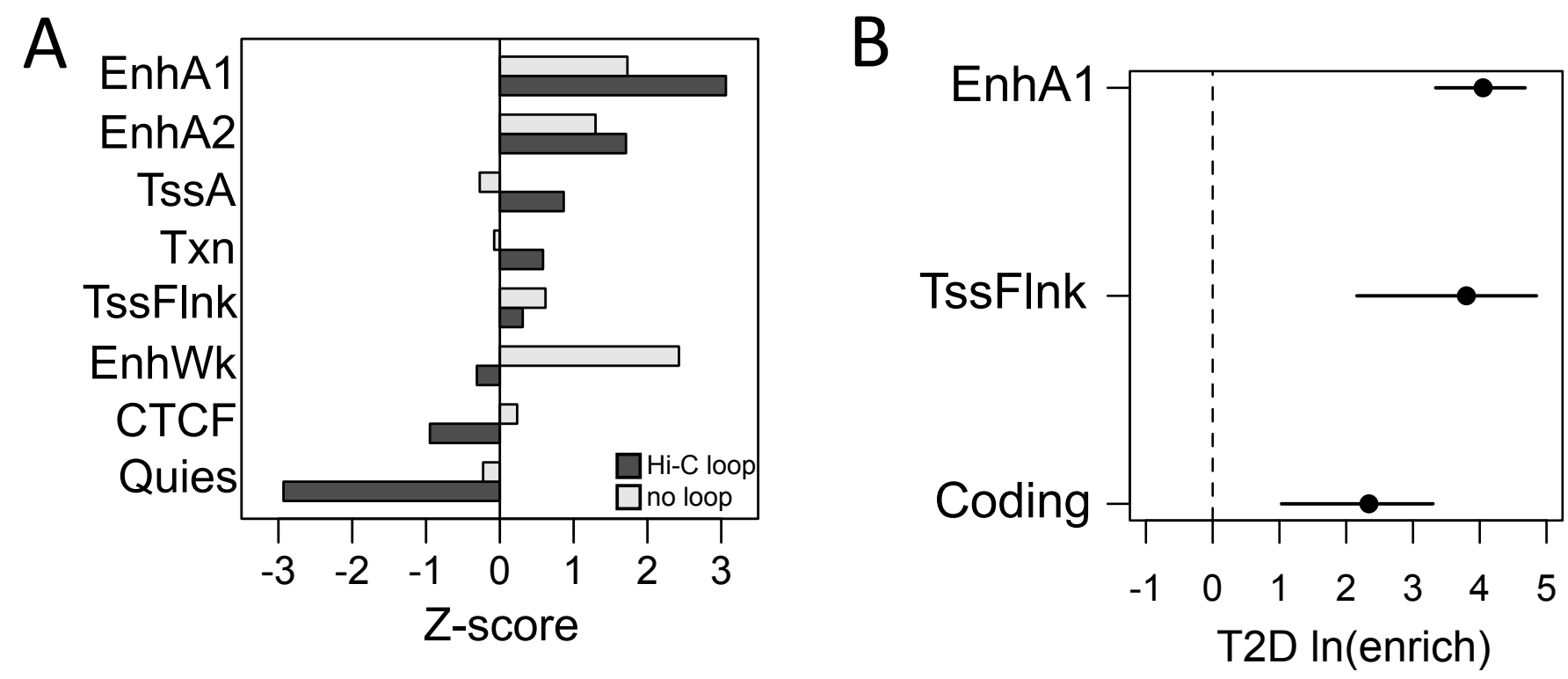

C

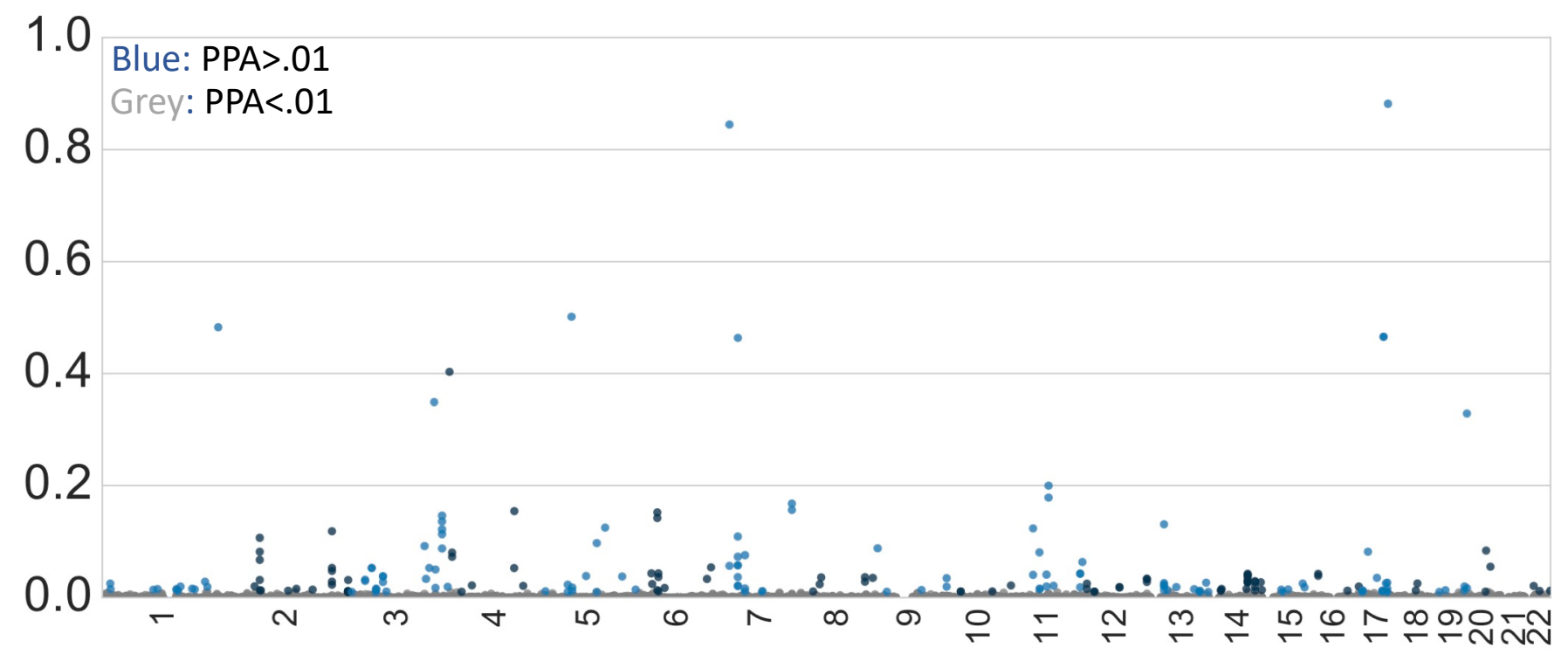

Figure S3. Effects of variants in pancreatic islet regulatory elements on T2D risk 
KCNQ1 locus

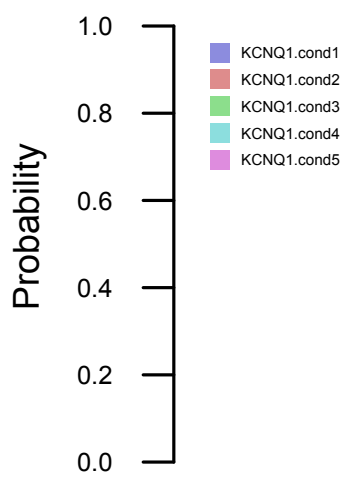

Hi-C loops

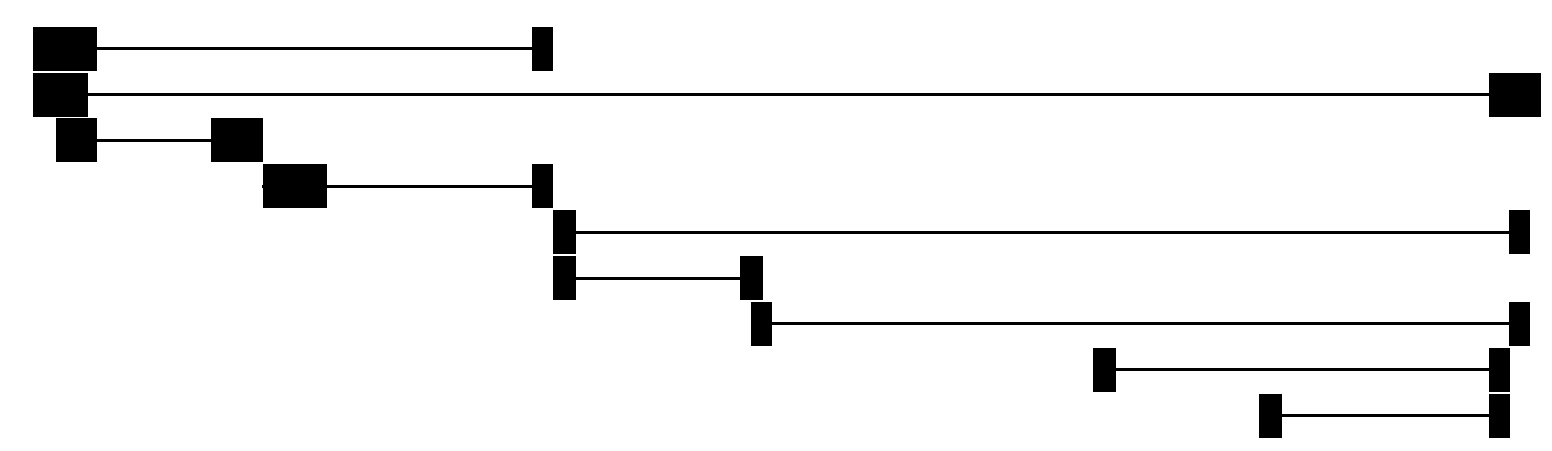

Chromatin state

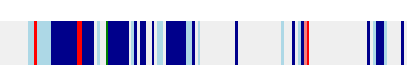

||||||| | |

| || ||| || | | |||| || || || ||I | ||

ATAC-seq

Genes

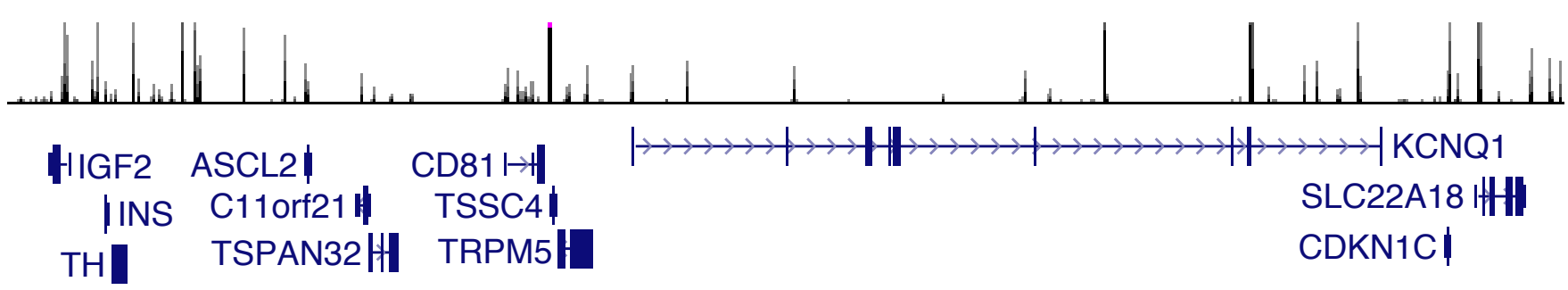

Figure S4. T2D enhancer signal chromatin loops to candidate target genes 
ZMIZ1 locus

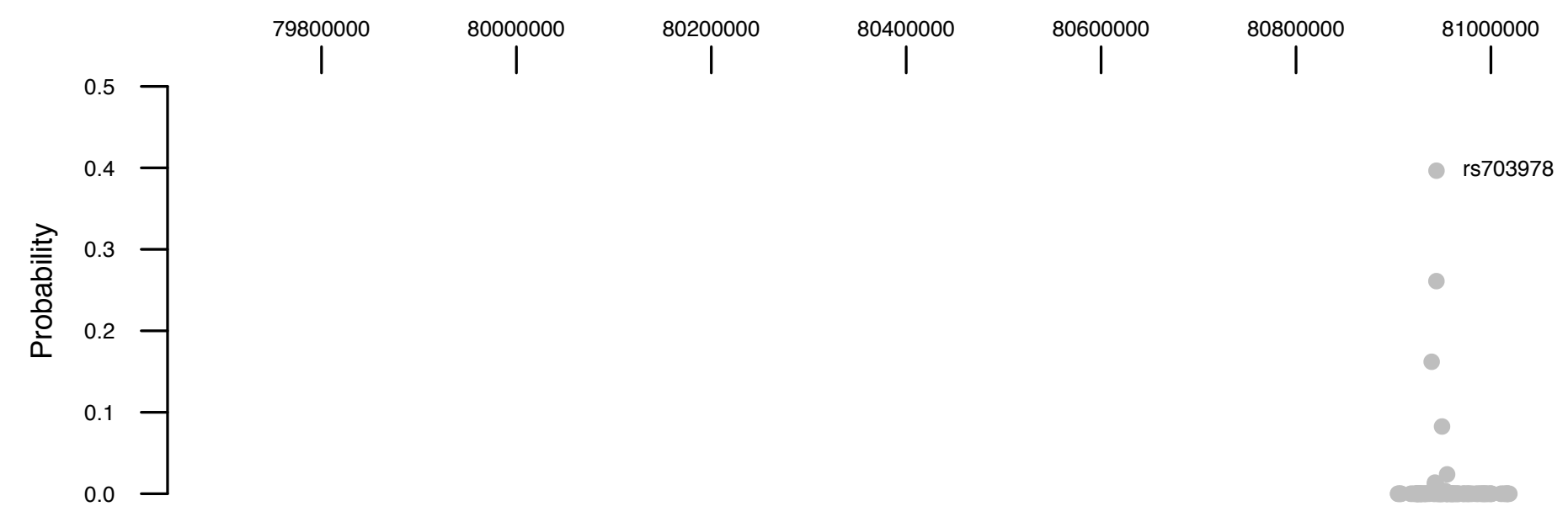

Hi-C loops
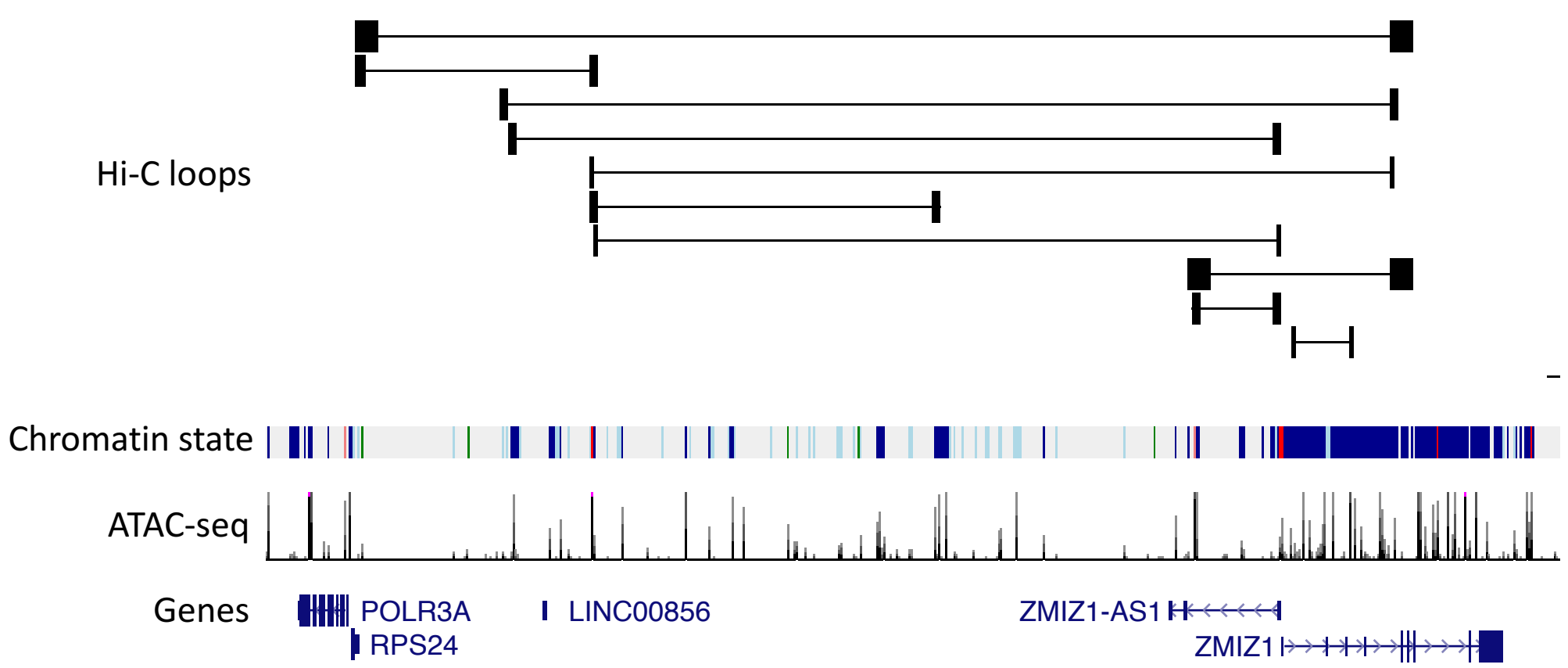

Figure S4. T2D enhancer signal chromatin loops to candidate target genes 
KCNJ11 locus

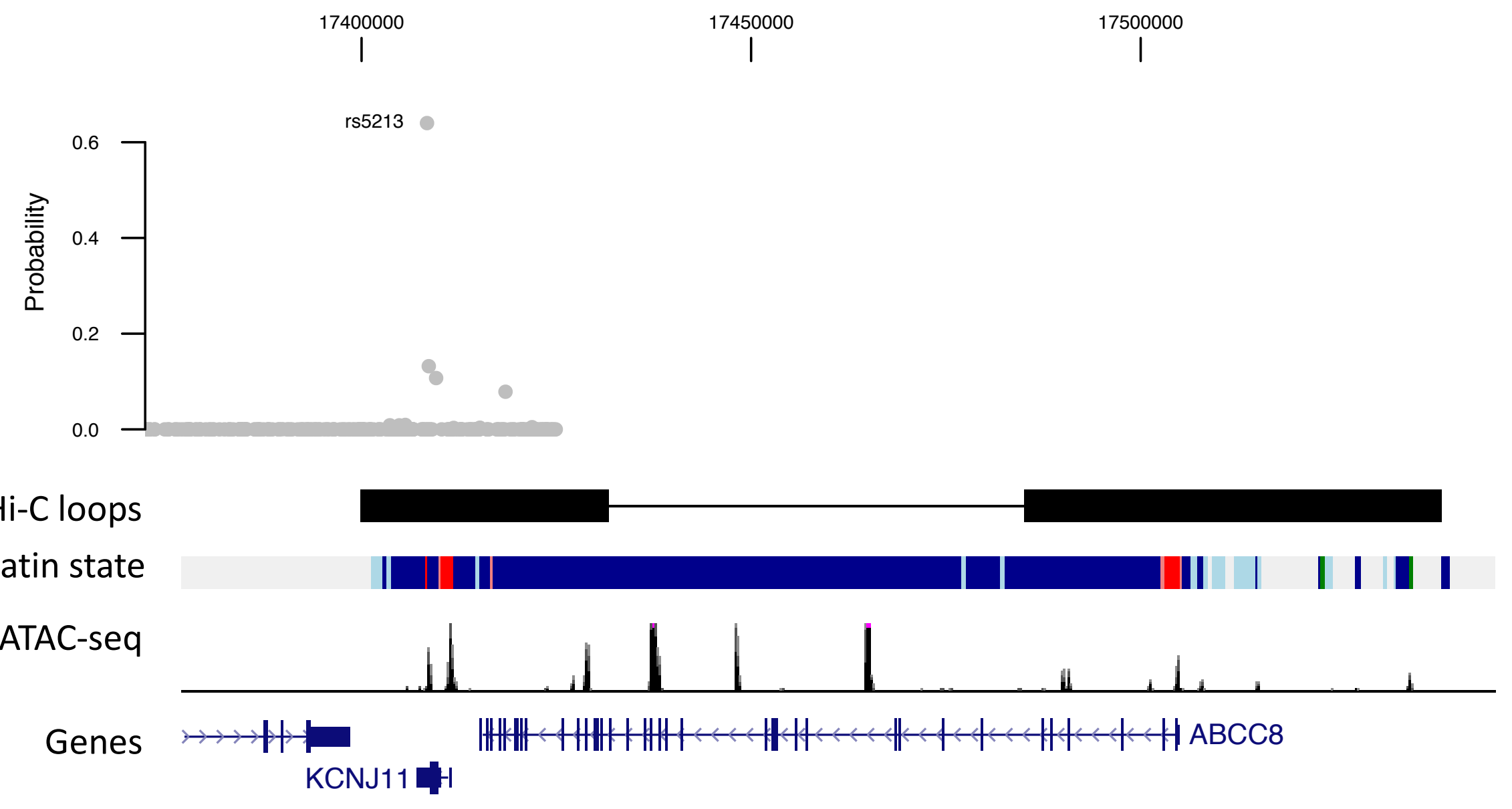

Figure S4. T2D enhancer signal chromatin loops to candidate target genes 
A

$14.12 \mathrm{~kb}$

After flp recombination:

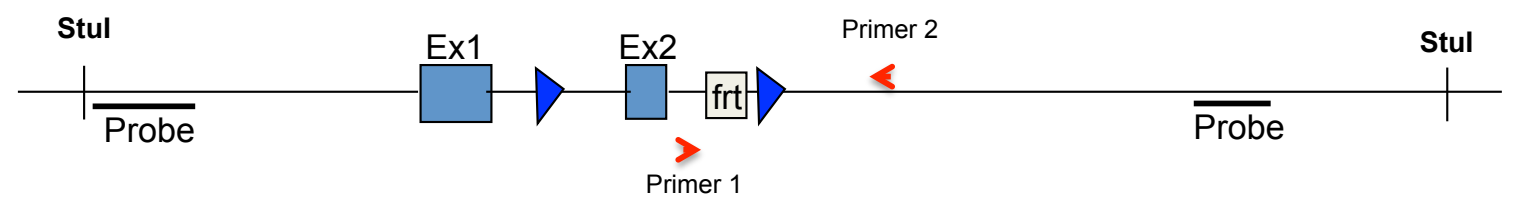

After Cre recombination:
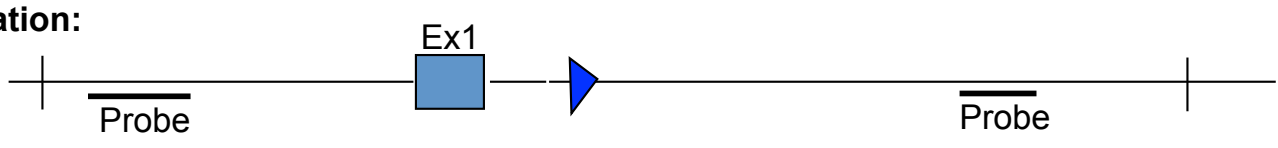

B

C
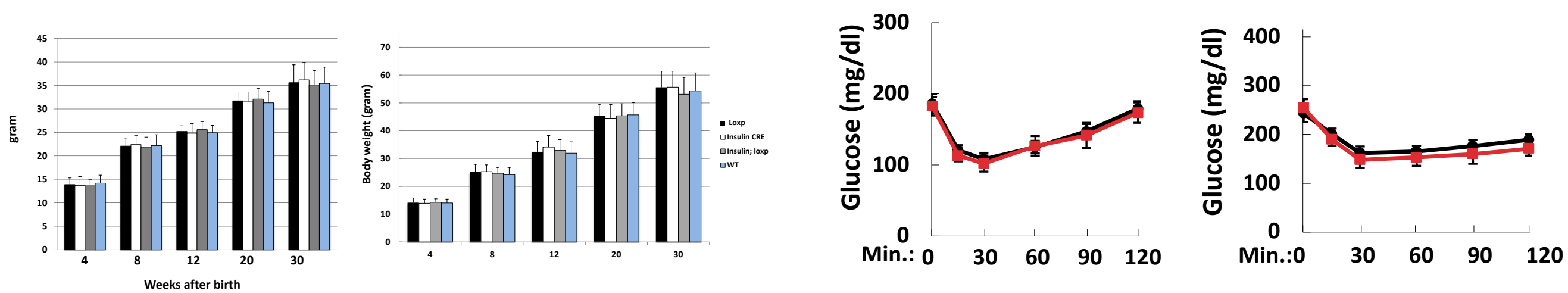

Figure S5. Characteristics of beta cell IMP2 mouse model 